\title{
Taming martensitic transformation via concentration modulation at nanoscale
}

Jiaming Zhu ${ }^{1,2}$, Yipeng Gao ${ }^{3}$, Dong Wang ${ }^{1}$, Tong-Yi Zhang ${ }^{4, *}$, Yunzhi Wang ${ }^{1,3, *}$

${ }^{1}$ Center of Microstructure Science, Frontier Institute of Science and Technology, Xi'an Jiaotong University, Xi'an 710049, China

${ }^{2}$ Department of Mechanical Engineering, Hong Kong University of Science and Technology, Clear Water Bay, Kowloon, Hong Kong, China

${ }^{3}$ Department of Materials Science and Engineering, The Ohio State University, 2041 College Road, Columbus, OH 43210, USA

${ }^{4}$ Shanghai University Materials Genome Institute and Shanghai Materials Genome Institute, Shanghai University, 99 Shangda Road, Shanghai 200444, China

\begin{abstract}
Martensitic transformation (MT) is typically a strongly first-order transition with autocatalysis in nucleation followed by rapid growth. It usually takes place within a narrow temperature or stress range, making its utilization in a controllable manner difficult. We show by computer simulations how MTs can be tailored by concentration modulation at the nanoscale in the parent phase, which induces spatial variations of both the stability of martensite and the transformation strain and tunes the overall MT kinetics from a typical first-order transition into a high-order like continuous transition. Such a unique MT characteristic reduces or even eliminates the transformation hysteresis and produces quasi-linear elasticity with ultra-low apparent elastic modulus.
\end{abstract}

Keywords: Concentration modulation, Martensitic transformation, Hysteresis, Pseudo-elasticity, Computer simulation 


\section{Introduction}

For an important class of smart materials, the shape memory alloys (SMAs), their functionalities (including shape memory effect and pseudo-elasticity) originate from martensitic transformations (MTs) [1,2]. MTs, being strongly first-order in nature [3], normally take place in an avalanche-like manner within a narrow temperature or stress range [4,5] and are accompanied by structural defects, large stress-strain and temperature-strain hysteresis and strongly nonlinear pseudo-elasticity, which leads to a variety of problems in applications [6,7]. For example, structural defects generate irrecoverable strain and cause functional fatigue and dimensional instability, which limit the service life of devices [7]. Even for the most widely used commercial SMA, $\mathrm{NiTi}$, the irrecoverable strain may approach $10 \%$ after 100 thermal cycles under a 150 MPa bias load [8], and failure by fracture would occur after a few thousand cycles [9]. In addition, the efficiency of SMA actuators is often less than $1 \%$ in practical applications due to large hysteresis, which is lower by several tens of times than that of piezoceramic actuators and hydraulic actuators [6]. Moreover, precise position control of actuators is required in micro-electromechanical systems, e.g., robotics and active catheters, but the strongly nonlinear pseudo-elasticity of SMAs makes such control difficult [10-12].

Extensive studies have been carried out lately to tailor the stress-strain or temperaturestrain responses and ameliorate degradation of SMAs. It has been shown that SMAs with high reversibility and low hysteresis can be developed by improving the geometrical compatibility between the parent and martensitic phases [13-17]. But the strict requirement on the transformation strain makes this approach applicable only to limited material systems (i.e. $\mathrm{Ti}-\mathrm{Ni}-\mathrm{Cu}-\mathrm{Pd}$ and $\mathrm{Zn}-\mathrm{Au}-\mathrm{Cu}$ ). Additionally, it has been shown theoretically that a giant non-hysteretic strain response could be achieved if the structural anisotropy of a low-symmetry phase vanishes [18], but experimental validation of this prediction has yet to come. Very recently, superelasticity with low hysteresis in a wide temperature range was found in $\mathrm{Fe}-, \mathrm{Co}-, \mathrm{Cr}-, \mathrm{Mn}$-doped $\mathrm{TiNi}$ system [19-21], Ni-rich TiNi alloys [22,23], Ni-Mn alloys [24] and AuCuAl alloys [25] under the assistance of strain glass transitions. Unfortunately, strain glass transitions are found so far only in ferroelastic systems having small transformation strains like the R phase in TiNi SMAs [26]. However, both experimental [20,23] and simulation $[19,26]$ studies on strain glass transitions have demonstrated that the 
hysteresis of MTs can be tuned if the sharp first-order transition could be converted into a broadly smeared high-order like transition and that functional fatigue and nonlinear pseudo-elasticity can be effectively inhibited by reducing the transition hysteresis [16,18,19,27].

It is well documented that MT is sensitive to alloy composition [2,28-30]. In nitinol, for example, one atomic percent variation in Ni concentration could change the MT start temperature $\left(\mathrm{M}_{\mathrm{s}}\right)$ by $\sim 100 \mathrm{~K}$ [30]. Similar phenomena are also found in other SMAs and gum metals, including TiNb [31,32], TiMo [33], AuCuZn [16], and CuAlbased [1] alloys. In addition to $\mathrm{M}_{\mathrm{s}}$, alloy composition may also have a strong effect on the transformation strain $[31,34,35]$. This suggests that concentration modulation (CM) in the parent phase could be an effective way of altering the nature of MTs. For example, it has been reported that the superelasticity and thermal expansion of Ti2448 (Ti-24Nb-4Zr-8Sn-0.10O in wt.\%) can be tuned by regulating the MT by CMs [36]. In Ti-Nb, there exist a large miscibility gap, with a spinodal range between $\sim 10$ to $\sim 90$ at.\% of $\mathrm{Nb}$ [37]. The hysteresis and stress window for the MT in this system are found to be sensitive to alloy composition and solution heat treatment schedule [31]. Additionally, the spinodally decomposed $\mathrm{Mn}-\mathrm{Cu}$ alloy is found to have a much higher $M_{s}$ than its uniform concentration counterpart dose and the $M_{s}$ shifts to higher temperatures with longer holding time during the spinodal decomposition [38,39]. As a matter of fact, CMs generated by spinodal decomposition are widely observed in SMAs, including TiNb [37,40], MnCu [38,39], TiV [41], FeMn [42] alloys, etc., with wavelengths ranging from several to several hundreds of nanometers [42]. Thus CMs generated via spinodal decomposition [43] in the parent phase could be utilized to alter the nature of MTs in SMAs. However, the detailed features of MTs in a compositionally modulated system remain largely unexplored.

In this study, we investigate by computer simulation how CMs at the nanoscale produced by spinodal decomposition in the parent phase affect the nature of MT in TiNb-based SMAs. We hypothesize that a spatial modulation in $\mathrm{M}_{\mathrm{s}}$ accompanying the CM will make the MT highly heterogeneous and occur in a broad temperature range, while a transformation strain modulation will lead to different geometrical compatibility between the parent and martensitic phases and hence different local stress states. The simulation results and analyses suggest that introducing spatial 
modulation of concentration in the parent phase at the nanoscale is indeed able to convert the otherwise strongly first-order MT into an overall (or macroscopically) continuous transformation with slim hysteresis, quasi-linear pseudo-elasticity with large work output, and ultra-low apparent elastic modulus. Furthermore, strong fatigue resistance and Invar and Elinvar anomalies [36,44] could also be expected because of the continuous nature of the MT.

\section{Phase field model}

\subsection{Symmetry breaking during MT in TiNb-based SMAs}

A phase filed model is formulated for the multifunctional $\beta$ TiNb-based alloys. According to the Burgers lattice correspondence (LC) [45] for the $\beta$ (BCC, point group $m \overline{3} m$ ) to $\alpha^{\prime \prime}$ martensite (orthorhombic, point group $\mathrm{mmm}$ ) transformation [46]

$$
[001]_{\beta} \rightarrow[100]_{\alpha "},[1 \overline{1} 0]_{\beta} \rightarrow[010]_{\alpha}{ }^{\prime \prime},[110]_{\beta} \rightarrow[001]_{\alpha}{ }^{\prime \prime}
$$

and the symmetry operations in the point groups that preserve such an LC, only 8 operations (out of the 48 symmetry operations in $m \overline{3} m$ ) [47] are left after the transformation, leading to $6(=48 / 8)$ crystallographic equivalent deformation modes (or correspondence variants) [48] characterized by 6 different transformation strain tensors. In fact, because an internal shuffle of $\{110\}_{\beta}$ atomic planes is also involved during the transformation [49-51], which doubles the deformation variants, there are 12 deformation variants in total [52]. However, an internal shuffle dose not contribute to the transformation strain [3]. Thus, if we choose the three orthogonal axes of the cubic crystal of the parent phase as the reference coordinate system, as shown in Fig. 1(a), and follow the Burgers correspondence, the transformation matrices that map the parent phase lattice onto that of the martensitic variants by a uniform affine deformation read

$$
\begin{gathered}
\boldsymbol{U}_{1}=\left[\begin{array}{ccc}
\zeta & 0 & 0 \\
0 & \frac{\alpha+\gamma}{4} & \frac{\gamma-\alpha}{4} \\
0 & \frac{\gamma-\alpha}{4} & \frac{\alpha+\gamma}{4}
\end{array}\right], \boldsymbol{U}_{2}=\left[\begin{array}{ccc}
\zeta & 0 & 0 \\
0 & \frac{\alpha+\gamma}{4} & \frac{\alpha-\gamma}{4} \\
0 & \frac{\alpha-\gamma}{4} & \frac{\alpha+\gamma}{4}
\end{array}\right], \\
\boldsymbol{U}_{3}=\left[\begin{array}{ccc}
\frac{\alpha+\gamma}{4} & 0 & \frac{\gamma-\alpha}{4} \\
0 & \zeta & 0 \\
\frac{\gamma-\alpha}{4} & 0 & \frac{\alpha+\gamma}{4}
\end{array}\right], \boldsymbol{U}_{4}=\left[\begin{array}{ccc}
\frac{\alpha+\gamma}{4} & 0 & \frac{\alpha-\gamma}{4} \\
0 & \zeta & 0 \\
\frac{\alpha-\gamma}{4} & 0 & \frac{\alpha+\gamma}{4}
\end{array}\right],
\end{gathered}
$$




$$
\boldsymbol{U}_{5}=\left[\begin{array}{ccc}
\frac{\alpha+\gamma}{4} & \frac{\gamma-\alpha}{4} & 0 \\
\frac{\gamma-\alpha}{4} & \frac{\alpha+\gamma}{4} & 0 \\
0 & 0 & \zeta
\end{array}\right], \boldsymbol{U}_{6}=\left[\begin{array}{ccc}
\frac{\alpha+\gamma}{4} & \frac{\alpha-\gamma}{4} & 0 \\
\frac{\alpha-\gamma}{4} & \frac{\alpha+\gamma}{4} & 0 \\
0 & 0 & \zeta
\end{array}\right] .
$$

where $\alpha=\sqrt{2} b / a_{0}, \zeta=a / a_{0}, \gamma=\sqrt{2} c / a_{0}$, with $a_{0}, a, b$ and $c$ being the lattice parameters of the parent and martensitic phase, respectively, as shown in Fig. 1(a). The corresponding stress-free transformation strain (SFTS) of the $p$-th variant, $\varepsilon_{i j}^{0}(p)$, can be calculated directly from the transformation matrices given in Eq. (1) according to the following equation

$$
\varepsilon_{i j}^{0}(p)=\frac{1}{2}\left(\boldsymbol{U}_{p}^{T} \boldsymbol{U}_{p}-\boldsymbol{I}\right),(p=1 \sim 6),
$$

where the superscript $T$ indicates matrix transpose, and $\boldsymbol{I}$ is the identity matrix.

Note that we are considering a compositionally non-uniform system in the current study and the lattice parameters of the parent and martensitic phases are functions of local concentration, which makes the SFTS in Eq. (2) a function of location as well. Both experimental measurements [31] and ab initio calculations [53] show that the concentration-dependence of lattice parameters of $\beta$ and $\alpha^{\prime \prime}$ phases in TiNb-based alloys can be approximated by linear functions (i.e., following the Vegard's law). Therefore in the simulations the variations of lattice parameters of $\beta$ and $\alpha^{\prime \prime}$ phases with concentration are obtained by fitting the data reported in the literature $[31,34,46,53,54]$ to linear functions, i.e.,

$$
\begin{gathered}
a_{0}=3.296+1.3 \times c_{N b} \times 10^{-4}, \\
a=2.894+1.2 \times c_{N b} \times 10^{-2}, \\
b=5.167-1.7 \times c_{N b} \times 10^{-2}, \\
c=4.748-4.8 \times c_{N b} \times 10^{-3},
\end{gathered}
$$

where $c_{N b}$ is the $\mathrm{Nb}$ concentration in atomic percent. Besides the SFTS, the $\mathrm{M}_{\mathrm{s}}$ also depends on the $\mathrm{Nb}$ concentration, which, according to [32,36,55], can be approximated by the following linear relationship

$$
M_{s}=550-24 \times c_{N b} .
$$

The dependence of the principle strains of the SFTS on Nb concentration is shown in Fig. 1(b), together with the variation of $\mathrm{M}_{\mathrm{s}}$. 


\subsection{Free energy formulation}

In our phase field approach, the parent and martensitic phases in TiNb-based alloys are characterized by using six non-conserved structural order parameters, $\eta_{p}(p=1 \sim 6)$, with $\left(\eta_{p=1 \sim 6}=0\right)$ representing the parent phase and $\left(\eta_{p}= \pm 1, \eta_{q=1 \sim 6, \text { but } q \neq p}=0\right)$ representing the $p$-th correspondence variant of the martensitic phase, where +1 and 1 denote atomic shuffles in two opposite directions in a correspondence variant. The total free energy functional, $F$, of the system is formulated as the following

$$
F=\int\left[\frac{1}{2} \kappa_{\eta} \sum_{p=1}^{6}\left(\nabla \eta_{p}\right)^{2}+f_{c h}\left(\eta_{1}, \cdots \eta_{6}\right)+f_{e x}\left(\eta_{1}, \cdots \eta_{6}\right)\right] d^{3} \boldsymbol{r}+E_{e l}
$$

where $\kappa_{\eta}$ is the gradient energy coefficient for structural non-uniformities following the gradient thermodynamics [56], $f_{c h}$ is the Landau free energy that describes the free energy of a local volume element having a uniform structural state characterized by $\eta_{p}(\boldsymbol{r})(p=1-6)$ [57-60]. During a MT that involves both shear and shuffle, either of them could be the primary transformation mode or, in many cases, the two modes are closely coupled together along the so-called minimum energy path (MEP) [61,62]. In the current study, we assume that the shuffle serves as the primary order parameter (i.e., $\eta_{p}$ ) parameterizing the MEP (i.e., serving as the reaction coordinate), then the stress-free transformation strain tensor for a local volume element located at $\boldsymbol{r}$ is given by

$$
\varepsilon_{i j}^{M T}(\boldsymbol{r})=\sum_{p=1}^{6} \varepsilon_{i j}^{0}(p, \boldsymbol{r}) \eta_{p}^{2}(\boldsymbol{r})
$$

and the Landau free energy can be approximated by the following polynomial [63]

$f_{c h}\left(\eta_{1}, \cdots \eta_{6}\right)=\frac{1}{2} A_{1}\left(T-T_{0}\right) \sum_{p=1}^{6} \eta_{p}^{2}(\boldsymbol{r})-\frac{1}{4} A_{2} \sum_{p=1}^{6} \eta_{p}^{4}(\boldsymbol{r})+\frac{1}{6} A_{3}\left(\sum_{p=1}^{6} \eta_{p}^{2}(\boldsymbol{r})\right)^{3}$,

where $A_{1}, A_{2}$ and $A_{3}$ are expansion coefficients. The $\mathrm{M}_{\mathrm{s}}$ modulation induced by the $\mathrm{CM}$ is incorporated into the model by formulating $T_{0}$ as a function of concentration, i.e., $T_{0}=493-24 \times c_{N b}$, following Eq. (4).

The free energy associated with an external stress, $\sigma_{k l}$, (i.e., the work term by the external stress) in Eq. (5), is given by the following equation 


$$
f_{e x}\left(\eta_{1}, \cdots \eta_{6}\right)=-\sigma_{k l} \cdot \varepsilon_{k l}^{M T}(\boldsymbol{r})
$$

The last term in Eq. (5), $E_{e l}$, is the coherency elastic strain energy of a structurally non-uniform but coherent system (i.e., martensitic variants coherently embedded in the parent phase matrix) characterized by $\eta_{p}(\mathbf{r})$. According to KhachaturyanShatalov's microelasticity theory (KS-theory) [64], it has the following close form

$$
E_{e l}=\frac{1}{2} \sum_{p=1}^{6} \sum_{q=1}^{6} \int \frac{d^{3} k}{(2 \pi)^{3}} B_{p q}(\boldsymbol{n})\left\{\eta_{p}^{2}\right\}_{k}\left\{\eta_{q}^{2}\right\}_{k}^{*}
$$

where the integral is taken in the reciprocal space, $\boldsymbol{n}=\frac{\boldsymbol{k}}{k}$ is a unit vector and $\boldsymbol{k}$ is a vector with modulus $k$ in the reciprocal space, $\left\{\eta_{p}^{2}\right\}_{k}$ represents the Fourier transform of $\eta_{p}^{2},\left\{\eta_{q}^{2}\right\}_{k}^{*}$ is the complex conjugate of $\left\{\eta_{q}^{2}\right\}_{k}$. Note that $\boldsymbol{k}=0$ is to be excluded from the integration. For a system with a free boundary, $B_{p q}(\boldsymbol{n})$ in Eq. (9) reads

$$
B_{p q}(\boldsymbol{n})= \begin{cases}0 \quad \boldsymbol{n}=0 \\ C_{i j k l} \varepsilon_{i j}^{0}(p, \boldsymbol{r}) \varepsilon_{k l}^{0}(q, \boldsymbol{r})-n_{i} \sigma_{i j}^{0}(p, \boldsymbol{r}) \Omega_{j k}(\boldsymbol{n}) \sigma_{k l}^{0}(q, \boldsymbol{r}) n_{l} \quad \boldsymbol{n} \neq 0\end{cases}
$$

where $\sigma_{i j}^{0}(p, \boldsymbol{r})=C_{i j k l} \varepsilon_{k l}^{0}(p, \boldsymbol{r})$ and $\Omega_{i j}^{-1}(\boldsymbol{n})=C_{i k l j} n_{k} n_{l}$.

\subsection{Coherency elastic strain energy in a compositionally non-uniform system}

It is noteworthy that $B_{p q}(\boldsymbol{n})$ in Eq. (10) is a continuous function of location in a compositionally non-uniform system due to the concentration-dependence of the transformation strain $\varepsilon_{i j}^{0}$. This means that $B_{p q}(\boldsymbol{n})$ needs to be calculated individually for each discrete point in the simulation, which is costly, especially for large three dimensional (3D) computational cells. To overcome this difficulty, we formulated a new equation for the coherency elastic strain energy in a compositionally non-uniform system

$$
E_{e l}=\frac{1}{2} \sum_{K=1}^{9} \sum_{L=1}^{9} \int \frac{d^{3} k}{(2 \pi)^{3}} B_{K L}^{n e w}(\boldsymbol{n})\left\{\theta_{K}\right\}_{k}\left\{\theta_{L}\right\}_{k}^{*},
$$

where $\left\{\theta_{K}\right\}_{k}$ represents the Fourier transform of $\theta_{K}$, which is defined as $\theta_{K}(\boldsymbol{r})=$ $\sum_{p=1}^{6}\left[\varepsilon_{i j}^{0}(p, r) \cdot \eta_{p}^{2}(\boldsymbol{r})\right]$ (the indices $i, j$ and $K$ in this expression are correlated via a generalized Voigt notation, i.e. $11 \rightarrow 1,22 \rightarrow 2,33 \rightarrow 3,23 \rightarrow 4,13 \rightarrow 5,12 \rightarrow 6,32$ $\rightarrow 7,31 \rightarrow 8,21 \rightarrow 9)$. Note that the transformation strain, $\varepsilon_{i j}^{0}(p, r)$, is a function of 
position because of its dependence on local concentration [31,34] (see Fig. 1(b)). For a system with a free boundary, $B_{K L}^{\text {new }}(\boldsymbol{n})$ in Eq. (11) reads

$$
B_{K L}^{n e w}(\boldsymbol{n})= \begin{cases}0 & \boldsymbol{n}=0 \\ C_{i j k l} & \varepsilon_{i j}^{\text {new }}(K) \varepsilon_{k l}^{\text {new }}(L)-n_{i} \sigma_{i j}^{\text {new }}(K) \Omega_{j k}(\boldsymbol{n}) \sigma_{k l}^{\text {new }}(L) n_{l} \quad \boldsymbol{n} \neq 0,\end{cases}
$$

where $\sigma_{i j}^{\text {new }}(K)=C_{i j k l} \varepsilon_{k l}^{\text {new }}(K), \Omega_{i j}^{-1}(\boldsymbol{n})=C_{i k l j} n_{k} n_{l}$, and

$$
\varepsilon_{k l}^{\text {new }}(K)=\left\{\begin{array}{ll}
1 & \text { if } K \text { is Voigt notation of } k l \\
0 & \text { otherwise }
\end{array} .\right.
$$

Note that such a boundary condition is required to simulate stress-controlled (constant stress) uniaxial tension.

\subsection{Kinetic equation}

The following time-dependent Ginzburg-Landau equation [65] is used to describe the temporal and spatial evolution of the structural order parameters during the MT

$$
\frac{d \eta_{p}(\boldsymbol{r}, t)}{d t}=-M \frac{\delta F}{\delta \eta_{p}(\boldsymbol{r}, t)}+\xi_{p}(\boldsymbol{r}, t), p=1 \sim 6,
$$

where $M$ is the kinetic coefficient and $\xi_{p}(\boldsymbol{r}, t)$ are the Langevin noise terms for structural fluctuations that meet the following fluctuation-dissipation theorem $[57,63]$ :

$$
\left\langle\xi_{p}(\boldsymbol{r}, t) \xi_{p}\left(\boldsymbol{r}^{\prime}, t^{\prime}\right)\right\rangle=2 \frac{k_{B} T}{|\Delta f| l_{0}^{3}} \delta\left(\boldsymbol{r}-\boldsymbol{r}^{\prime}\right) \delta\left(t-t^{\prime}\right)
$$

where $k_{B}$ is the Boltzmann constant, $T$ is the absolute temperature, $|\Delta f|$ is the chemical driving force (free energy difference between the parent and martensitic phases calculated from the Landau free energy), $l_{0}$ is the length scale of the computational grid increment, and $\delta$ is the Kronecker delta function.

Equation (13) is solved numerically in the reciprocal space using a finite difference method for a compositionally modulated model system. The total free energy of the parent phase at $773 \mathrm{~K}$ (a temperature above $\mathrm{M}_{\mathrm{s}}$ ) is formulated based on the $\mathrm{Ti}-\mathrm{Nb}$ binary system [56,66-68] in our spinodal decomposition model,

$$
F^{S D}=\int_{V}\left\{f(c)+\frac{\kappa_{c}}{2}(\nabla c)^{2}\right\} d V
$$


where $f(c)$ is the local chemical free energy, $c$ is $\mathrm{Nb}$ concentration, and $\kappa_{c}$ is the gradient energy coefficient. The dimensionless local chemical free energy $f^{*}(c)$ is approximated by a double-well function with the equilibrium concentrations of the two coexisting phases being 0.08 and 0.2 [36], respectively, e.g., $f^{*}(c)=$ $(c-0.08)^{2}(0.2-c)^{2}$. The dimensionless gradient energy coefficient is chosen to be $\kappa_{c}^{*}=0.691$. The time step $\Delta t^{*}$ is chosen to be 0.001 . The interfacial energy between the solute-rich and solute lean phases at equilibrium is assumed to be $\sim 0.01 \mathrm{~J} / \mathrm{m}^{2}$, which yields a numerical grid size of $l_{0} \sim 1 \mathrm{~nm}$. The temporal evolution of the concentration field is governed by the Cahn-Hilliard equation [69].

\subsection{Model parameters}

The elastic constants of the parent phase, $\mathrm{C}_{11}=57.2 \mathrm{GPa}, \mathrm{C}_{12}=36.1 \mathrm{GPa}$ and $\mathrm{C}_{44}=35.9$ GPa are obtained from the literature [46,70]. They are assumed to be independent of concentration. Furthermore, homogeneous modulus assumption is adopted in this study, i.e., the $\alpha^{\prime \prime}$ martensitic phase is assumed to have the same elastic constants as that of the parent phase because of the lack of consistent experimental data for the elastic constants of $\alpha^{\prime \prime}$ martensites [5]. The expansion coefficients in the Landau free energy, $A_{1}=1.3 \times 10^{5} \mathrm{~J} / \mathrm{m}^{3}, A_{2}=11.74 \times 10^{7} \mathrm{~J} / \mathrm{m}^{3}, A_{3}=17.39 \times 10^{7} \mathrm{~J} / \mathrm{m}^{3}$, are chosen in such a way that the phase field model (with a uniform $\mathrm{Nb}$ concentration of 15 at.\%) produces a stress-strain curve that is consistent with the experimental result of Ti2448 that has the same $\mathrm{Nb}$ concentration [70], including the critical stress for the MT, stress hysteresis and superelasticity (see Fig. 2). The gradient energy coefficient for the structural non-uniformity is assumed to be $\kappa_{\eta}=1.2 \times 10^{-12} \mathrm{~J} / \mathrm{m}$, which yields an interfacial energy between the parent and martensitic phases of $\Gamma_{A M} \approx 0.01 \mathrm{~J} / \mathrm{m}^{2}$ (which falls in the range of the experimental data [71]) and a twin boundary energy between different martensitic variants of $\Gamma_{M M} \approx 0.02 \mathrm{~J} / \mathrm{m}^{2}\left(\Gamma_{M M} \approx 2 \Gamma_{A M}\right.$ according to reference [19]) with a length scale of $l_{0} \sim 1 \mathrm{~nm}$. The structural mobility in Eq. (13) is chosen as $M=1 \times 10^{-4} \mathrm{~m}^{4} \mathrm{~J}^{-1} \mathrm{~s}^{-1}$, estimated from $v=M(-\Delta f)[19,72]$, where $v$ is the interface velocity between the parent and martensitic phases during the MT $\left(\sim 10^{3} \mathrm{~m} / \mathrm{s}\right)$ and $\Delta f=-10^{7} \mathrm{~J} / \mathrm{m}^{3}[73,74]$ is the typical driving force for the MT. The amplitude of the Langevin noise is $2 \frac{k_{B} T}{|\Delta f| l_{0}^{3}}=0.3$ in our simulations at $300 \mathrm{~K}$. The system size used 
in the simulations is $128 l_{0} \times 128 l_{0} \times 128 l_{0}$. Periodical boundary conditions are applied along all three dimensions.

\section{Simulation results}

Figure 3(a) shows the simulation results of $\mathrm{CM}$ of $\mathrm{Nb}$ in a single-crystal (with an average $\mathrm{Nb}$ concentration of $15 \mathrm{at} . \%$ ) produced by spinodal decomposition in the parent $(\beta, \mathrm{BCC})$ phase of our model system. The one-dimensional (1D) concentration wave along the body-diagonal of the computational cell and the statistical distribution of voxels with different $\mathrm{Nb}$ concentrations in the computational cell as function of aging time are shown in Fig. 3(b) and (c), respectively. The wavelength of the CM shown in Fig. $3(\sim 45 \mathrm{~nm})$ is reasonable for Ti-based alloys whose wavelength of spinodal decomposition could be several tens of nanometers $[42,75]$. It is readily seen from Fig. 3(a) that the $\mathrm{Nb}$-lean and $\mathrm{Nb}$-rich regions form a typical interpenetrated domain structure normally seen in spinodal decomposition with similar equilibrium volume fractions of the two phases.

Being concentration-dependent, both $\mathrm{M}_{\mathrm{s}}$ and the transformation strain modulate in space accompanying the $\mathrm{CM}$ in the system. This leads to drastically different stress strain (SS) curves (in terms of the hysteresis and critical stress for the MT, and the pseudo-elastic behavior) for systems having different CMs (in terms of wavelength and amplitude), as shown in Fig. 3(d). Before aging (i.e. $\mathrm{t}^{*}=0$ ), the SS curve in Fig. 3(d1) shows a large stress hysteresis and an obvious stress plateau (i.e. strongly nonlinear pseudo-elasticity) that are typical characteristics of conventional MTs widely observed in experiments. However, the SS curve changes gradually to slim ones with narrow hystereses as the CM in the parent phase develops, as shown in Fig. 3(d2)-(d4). In particular, as compared to Fig. 3(d1), the hysteresis and $\sigma_{\mathrm{Ms}}$ are reduced drastically and the stress plateau disappears completely (Figs. 3(d3) and (d4)).

Being consistent with the variation of the SS curve, the volume fraction of martensite vs. stress curve shows a similar transition from a square-like loop (sharp changes) (Fig.3 (e1)) to a slim and smooth loop (gradual changes) (Fig. 3(e3) and (e4)), again demonstrating a transition from a sharp first-order MT to a higher-order like continuous transition. 
However, it is noteworthy that the stress plateau reemerges in Fig. 3(d5). Correspondingly, the volume fraction of martensite shows a sharp jump (from 0 to 0.27) in Fig. 3(e5), which is similar to but smaller than the jump (from 0 to 0.98 ) shown in Fig. 3(e1). The martensitic volume fraction of 0.27 is close to the total volume fraction $(\sim 0.28)$ of voxels in the computational cell that have $\mathrm{Nb}$ concentration of 8 10 at.\% (as shown in Fig. 3(c5)). This indicates that the stress plateau and volume fraction jump are caused by MT in regions having a $\mathrm{Nb}$ concentration of 8 10 at.\%. Since the CM wavelength in Fig. 3(a5) is approaching to the size of the computational cell, domains having $\mathrm{Nb}$ concentration of $8 \sim 10$ at.\% are rather large and thus the MT in these regions leads to a macroscopic strain burst in Fig. 3(d5). In addition, an open-loop strain is observed in Fig. 3(d5), which corresponds to retained martensitic domains shown in Fig. 3(e5).

\section{Discussion}

\subsection{Influence of CM on MT}

The pseudo-elastic behavior, as a mechanical response of internal microstructure in a ferroelastic system to an external load, is determined by microstructure evolution during forward and backward MTs. In contrast to a compositionally uniform system, the stability of martensite and thus $\mathrm{M}_{\mathrm{s}}$ modulate following the $\mathrm{CM}$ in a compositionally non-uniform system, which alters the nature of the MT drastically. Take the MT of the system shown in Fig. 3(a3) as an example. Upon loading, martensitic particles of preferred variants first appear in the Nb-leanest regions (highlighted in green in Fig. 4(j)) where the $\mathrm{M}_{\mathrm{s}}$ is the highest according to Fig. 1(b) (see, e.g., the particle indicated by an arrow in Fig. 4(a)). Then these martensitic particles grow gradually toward Nb-richer regions with increasing external load (see Fig. 4(b)-(c) and a zoom-in image in Fig. 4(k)), accompanied by the formation of new martensitic particles in Nb-richer regions as well. At the end of loading, most of the computational cell is occupied by martensite (see Fig. 4(d), where the volume fraction of the martensite reaches 0.89 ), with retained parent phase in regions of the lowest $\mathrm{M}_{\mathrm{s}}$ (i.e., regions of the highest $\mathrm{Nb}$ concentration, as indicated by the orange color in Fig. 4(j) and marked by the orange circle in Fig. 4(d)). During unloading, the martensite transforms back to the parent phase starting from the $\mathrm{Nb}$-richest regions, following a reverse sequence of the loading process (see Figs. 4(e)-(g) and a zoom-in image in 
Fig. 4(l)), and disappears completely when the applied load vanishes (Fig. 4(h)). Note that only variants $3,4,5$ and 6 are observed during the loading and unloading process shown in Fig. 4(a)-(h). This is because only these four variants are preferred by the tensile load applied along the $[100]_{\beta}$ direction, as shown in Fig. 5.

Thus the nucleation and growth (upon loading) and shrinkage (upon unloading) of martensitic domains (Fig. 3(e3) and (e4)) all occur continuously and gradually in a compositionally non-uniform system with $\mathrm{CM}$ of certain wavelength, which are in sharp contrast to the avalanche-like discontinuous manner of conventional forward and backward MTs (Fig. 3(e1)) in a compositionally uniform system. The continuous nature of the progress in both forward and backward MTs originates from (i) a broad distribution of phase stability of martensite or $M_{s}$ in the system associated with the $\mathrm{CM}$ and (ii) spatial confinement directly from the CM that has a wavelength much smaller than the normal micrometer-sized internally twinned martensitic plates or herringbone structures. As shown in Fig. 6(a), the martensite could be stable, metastable or unstable depending on the local $\mathrm{Nb}$ concentration (Fig. 6(b)) and these regions interpenetrate each other in space, imposing strong confinement upon each other. Even though there are a few spots in the system where the $\mathrm{Nb}$ concentration reaches 9.3 at\% (Fig. 6(b)) and, according to Fig. 6(a), the martensitic phase is stable in these regions, the Langevin noises introduced in Eq. (13) cannot induce any nucleation event during the simulation without the aid of external loads. Thus with the assistance of an external load, the MT occurs locally within regions of the lowest $\mathrm{Nb}$ concentration first and more and more sites are activated for the MT as the load increases. However, the interpenetrating spatial confinement from regions of higher $\mathrm{Nb}$ concentrations (thus higher parent phase stability) suppresses completely the autocatalysis of nucleation and confines strictly the extent of growth. Because of the broad $\mathrm{M}_{\mathrm{s}}$ distribution in the system, the MT starts locally at a much lower stress level as compared to that found in the compositionally uniform system (Fig. 3(d1)) and the MT continues to "harden" as the "easier to transform" regions are gradually exhausted. Because of the existence of highly stable parent phase regions (e.g., $C_{5}$ in Fig. 6), retained parent phase exist in the system even at the end of loading. Thus, no nucleation of the parent phase is involved during the reverse transformation upon unloading. As a consequence, the otherwise avalanche-like MT is converted into a broadly smeared and overall continuous transition and the stress hysteresis 
accompanying the MT is dramatically reduced, as shown in Fig. 3(d).

Even though the above discussions are made for stress induced MT in a compositionally non-uniform system with $\mathrm{CM}$ of a given wavelength $(\sim 45 \mathrm{~nm}$, Fig. 3(a3)), similar phenomena and MT characteristics are expected for thermally induced MT. Figure 7 shows the simulation results obtained by thermal cycling of the spinodally decomposed parent phase shown in Fig. 3(a3). Similar to the case shown in Fig. 3(e3), the volume fraction of the martensite increases or decreases gradually as temperature decreases or increases (Fig. 7(i)). The evolution of the martensitic domain structures occurs within a broad temperature range during cooling and heating (Fig. 7(a)-(h)), which is also similar to that observed in the stress-induced MT. However, there is one obvious difference, i.e., all the martensitic variants appear during this thermally induced MT (Fig. 7(a)-(h)), which leads to the obvious differences in colors of the martensitic domain structures shown in Fig. 4 and Fig. 7.

It should be pointed out that no thermally induced MT is observed experimentally in the concentration modulated Ti2448 with a concentration wavelength of $\sim 6 \mathrm{~nm}$ [36]. Our simulation results suggest that the thermally induced MT could occur in Ti2448 if the $\mathrm{CM}$ wavelength is increased significantly by aging. It will be shown below (Section 4.3) that the CM wavelength does have a strong influence on MTs in a compositionally non-uniform system.

\subsection{Influence of CM on mechanical properties}

From the SS curve, one can extract useful information about the mechanical behavior of the system. For example, Fig. 8 shows the variation of $\sigma_{\mathrm{Ms}}$, area of the stress hysteresis loop and the tangent modulus determined from the SS curve. The value of $\sigma_{\mathrm{Ms}}$ is determined by the tangent method shown in the inset of Fig. 8(a) while the tangent modulus is defined as the slope of the transformation hardening region as shown in the inset of Fig. 8(b). It is readily seen that these properties can be tuned effectively by changing the $\mathrm{CM}$ in the parent phase through aging. Therefore, it is expected that a combination of low hysteresis, low apparent Young's modulus and quasi-linear elasticity could be achieved in a single SMA via CM engineering. The transformation hardening effect is one of the distinct features of the CM system, which prevents the system from losing mechanical stability during the MT. This also 
provides a promising way to reduce the apparent Young's modulus via MT without sacrificing strength [76]. As Fig. 8(b) shows, the tangent modulus of the alloy is actually lower than the Young's modulus of human bones (20 30GPa) and can be tuned easily by aging. Thus composite materials could be designed [44] to have the concentration modulated SMAs strained beyond $\sigma_{\mathrm{Ms}}$ in the composites to match the apparent modulus of the composite with that of human bones.

\subsection{Effect of CM wavelength}

Figures 3(d1)-(d4) show the disappearance of the stress plateau when the CM is introduced in the parent phase. However, the stress plateau reemerges (Fig. 3(d5)) when the aging time and thus the CM wavelength exceeds certain values (Fig. 3(a5)). The microstructure evolution process during the stress-induced MT of the CM system shown in Fig. 3(a5) is shown in Fig. 9 together with the SS curve. Note that the parent phase is not transparent anymore but represented by the green color in this plot. It is readily seen from Figs. 9(b) and (c) and their locations on the SS curve that the stress plateau is caused by the burst of a few relatively large internally twinned martensitic domains that take up a considerable volume fraction of the system as shown in Fig. 3(e5). The sudden appearance of a large amount of martensitic domains is made possible by the existence of relatively large $\mathrm{Nb}$-lean regions in the initial microstructure (associated with the much longer CM wavelength) (see the inset of Fig. 9(i) and Fig. 3(a5)). In addition, an open-loop strain is observed in Fig. 9(i) which is caused by the residual martensite shown in Fig. 9(h) because the martensitic phase is stable in regions with a $\mathrm{Nb}$ concentration of $\sim 8$ at.\% at the simulation temperature 300 $\mathrm{K}$ [76]. The SS curve of a system with a uniform $\mathrm{Nb}$ concentration of 8 at.\% is shown Fig. 10 for comparison. Thus, when the wavelength of the $\mathrm{CM}$ in the parent phase becomes much larger than the domain size of internally twinned martensitic plates, the CM loses its confinement effect and the MT becomes similar to that in a compositionally uniform system.

On the other hand, if the CM wavelength is much smaller than stable internally twinned martensitic domains, the system will behave similar to a homogeneous system, as shown by the SS curves in Fig. 11. In this system the parent phase has a $\mathrm{CM}$ of a wavelength of $\sim 6 \mathrm{~nm}$. Note that the computation cells in Fig. 3 and Fig. 11 have the same average $\mathrm{Nb}$ concentration of $15 \mathrm{at} . \%$. Therefore, the effectiveness of 
CM in taming MT depends on the relative size between the martensitic domains and the CM wavelength. The critical size of an internally twinned martensitic domain is determined by the interplay between the coherency elastic strain energy and the interfacial energy (including twin-boundary energy and parent-martensite interfacial energy) for a fixed chemical driving force for the MT.

It should be pointed out that the Ti2448 single-crystal shows a large stress hysteresis and strongly nonlinear pseudo-elasticity [70] (see Fig. 2). However, its polycrystalline counterpart has relatively low stress hysteresis and quasi-linear pseudo-elasticity [77]. Our simulation studies show that different CMs in the parent phase crystals may have strong influence on the MT and hence the mechanical response of systems, which may shed light on the difference in the mechanical properties between the single- and poly-crystals of Ti2448.

\subsection{Comparison with strain glass transition}

Strain glass transition (SGT) in doped ferroelastic systems has also been shown to be able to change a sharp, discontinuous MT into a broadly smeared one [19,20,22,23]. The microstructural evolution during a SGT is characterized by the formation of nanodomains of individual martensitic variants in the absence of any long-range strain order $[4,20,23,78]$, which is quite different from the internally twinned martensitic domains observed in the current study (see, e.g., Fig. 3). It is believed that the physical origin of the STG is the local stress field generated by randomly distributed point defects that prevents the formation of long-range order strain patterns $[4,79]$. Because of the relatively small local strains associated with the point defects as compared to the SFTS of MT, the STG mechanism operates only in systems with relatively small SFTS [26] like the R martensite in NiTi alloys and thus with limited work output. In contrast, Figures 3(d)-(e) demonstrate clearly that a CM system can generate a broadly smeared MT with large SFTSs, which has not only low hysteresis and quasi-linear elasticity, but also large work output. The physical origin of the broadly smeared MT in a CM system is the $\mathrm{M}_{\mathrm{s}}$ modulation accompanying the $\mathrm{CM}$ and this mechanism is more effective in systems having strong concentration dependent of $\mathbf{M}_{\mathrm{s}}$, which has been observed widely in experiments [1,16,31-33,38,39,76]. Note that CMs at the nanometer scales can be achieved not only by spinodal decomposition $[39,80-83]$ but also by nano-precipitates in the parent phase under under-aged 
conditions, i.e., growing precipitates with their diffusion fields just start to overlap [84-87]. Thus the current study suggests ample opportunities for developing novel SMAs with slim hysteresis, quasi-linear pseudo-elasticity with large work output, ultra-low apparent elastic modulus and Invar and Elinvar anomalies [19,36,88,89]. It also shows that the SGT is not a necessary condition to produce broadly smeared MTs.

\subsection{Effect of geometrical compatibility variation}

In $\mathrm{CM}$ systems, since the lattice parameters are function of concentration, the geometrical compatibility between the parent and martensitic phases should vary with the CMs, and so should the lattice correspondence and orientation relationship. In the system considered in the current study, the Nb-rich regions have better geometrical compatibilities in terms of the cofactor condition [90], because in regions of 17.4at.\% $\mathrm{Nb}$ one of the principle strain, $\lambda_{3}$, is close to zero as shown in Fig. 1(a). Moreover, the other two principle strains, $\lambda_{1}$ and $\lambda_{2}$, have smaller values in magnitude in the Nb-rich regions. This varying compatibility effect has been captured in our simulations as well because the location-dependence of the SFTS has been considered in our new elastic energy formulation for a compositionally non-uniform system (see Eqs. (11) and (12)).

\section{Summary}

Both stress- and temperature-induced martensitic transformations (MTs) and the corresponding mechanical properties in compositionally non-uniform TiNb-based SMAs are investigated systematically by computer simulations using the phase field method. Concentration modulations (CMs) with different wavelengths at the nanoscale are first generated by spinodal decomposition at different stages in the parent phase. Both the stress-free transformation strain (SFTS) and the MT start temperature $\left(\mathrm{M}_{\mathrm{s}}\right)$ are assumed to be strong function of concentration according to available experimental data. To calculate the coherency elastic strain energy of MT in such concentration modulated systems, a new mathematical formulation that accounts for spatially varying SFTS is derived. The simulation results show that the continuous $\mathrm{Nb}$ concentration variation and the interpenetration of $\mathrm{Nb}$-lean and $\mathrm{Nb}$-rich domains in $3 \mathrm{D}$ lead to continuous variation of phase stability and $\mathrm{M}_{\mathrm{s}}$ and the interpenetration of high $\mathrm{M}_{\mathrm{s}}$ and low $\mathrm{M}_{\mathrm{s}}$ regions at the nanoscale in the concentration modulated 
systems. Such unique heterogeneous parent phase microstructures regulate strictly the MT (both nucleation and growth) during either stress or temperature cycling, suppress completely autocatalysis and limit the extent of growth at each given stress or temperature increment. Overall, it changes the nature of the MT from sharp, discontinuous, strongly first-order (expected for a uniform system) to broadly smeared, continuous and high-order like. Correspondingly, the transformation hysteresis is reduced drastically, the strongly nonlinear pseudo-elasticity becomes a quasi-linear one (within a rather large strain range as compared to that achieved by the strain glass transitions), and ultra-low apparent elastic modulus. Because of the continuous nature of the overall MT found in these concentration modulated systems, the Invar and Elinvar anomalies are also expected for these alloys. Opportunities to engineer composite SMA systems with apparent elastic modulus matching that of human bones are also suggested.

\section{Acknowledgement}

JM Zhu acknowledges the financial supports from the Joint School of XJTU and HKUST and from the Hong Kong Research Grants Council under the General Research Fund (Project number, 622911); YG and YW acknowledge the financial supports of the US Natural Science Foundation Grant No. DMR-1410322 and the US Department of Energy Grant No. DE-SC0001258; TY Zhang acknowledges the financial support of research grants (No. 15DZ2260300 and No. 16DZ2260600) from the Science and Technology Commission of Shanghai Municipality. D Wang acknowledges the National Key Research and Development Program of China (Grants No. 2016YFB0701302, 2014CB644003) and the National Natural Science Foundation of China (Grants No. 51671156), IRT13034.

\section{Reference}

[1] C. Lexcellent, Shape-memory Alloys Handbook, John Wiley \& Sons, Inc., Hoboken, NJ, USA, 2013.

[2] K. Otsuka, X. Ren, Physical metallurgy of Ti-Ni-based shape memory alloys, Prog. Mater. Sci. 50 (2005) 511-678. 
[3] J.W. Christian, The Theory of Transformations in Metals and Alloys, Elsevier, Oxford, UK, 2002.

[4] D. Wang, Y. Wang, Z. Zhang, X. Ren, Modeling Abnormal Strain States in Ferroelastic Systems: The Role of Point Defects, Phys. Rev. Lett. 105 (2010) 205702.

[5] J. Zhu, H. Wu, D. Wang, Y. Gao, H. Wang, Y. Hao, R. Yang, T.-Y. Zhang, Y. Wang, Crystallographic analysis and phase field simulation of transformation plasticity in a multifunctional $\beta$-Ti alloy, Int. J. Plast. 89 (2017) 110-129.

[6] J. Mohd Jani, M. Leary, A. Subic, M.A. Gibson, A review of shape memory alloy research, applications and opportunities, Mater. Des. 56 (2014) 10781113.

[7] G. Eggeler, E. Hornbogen, A. Yawny, A. Heckmann, M. Wagner, Structural and functional fatigue of NiTi shape memory alloys, Mater. Sci. Eng. A. 378 (2004) 24-33.

[8] M.L. Bowers, Y. Gao, L. Yang, D.J. Gaydosh, M. De Graef, R.D. Noebe, Y. Wang, M.J. Mills, Austenite grain refinement during load-biased thermal cycling of a Ni49.9Ti50.1 shape memory alloy, Acta Mater. 91 (2015) 318-329.

[9] R.D. James, Taming the temperamental metal transformation, Science (80-. ). 348 (2015) 968-969.

[10] J. Jayender, R.V. Patel, S. Nikumb, M. Ostojic, Modeling and Control of Shape Memory Alloy Actuators, IEEE Trans. Control Syst. Technol. 16 (2008) 279287.

[11] A.T. Tung, B.-H. Park, D.H. Liang, G. Niemeyer, Laser-machined shape memory alloy sensors for position feedback in active catheters, Sensors Actuators A Phys. 147 (2008) 83-92.

[12] B. Selden, K. Cho, H.H. Asada, Segmented shape memory alloy actuators using hysteresis loop control, Smart Mater. Struct. 15 (2006) 642-652.

[13] Z. Zhang, R.D. James, S. Müller, Energy barriers and hysteresis in martensitic phase transformations, Acta Mater. 57 (2009) 4332-4352.

[14] J. Cui, Y.S. Chu, O.O. Famodu, Y. Furuya, J. Hattrick-Simpers, R.D. James, A. Ludwig, S. Thienhaus, M.Wuttig, Z. Zhang, I. Takeuchi, Combinatorial search of thermoelastic shape-memory alloys with extremely small hysteresis width, Nat. Mater. 5 (2006) 286-290.

[15] R. Zarnetta, R. Takahashi, M.L. Young, A. Savan, Y. Furuya, S. Thienhaus, B. Maaß, M. Rahim, J. Frenzel, H. Brunken, Y. Chu, V. Srivastava, R.D. James, I. Takeuchi, G. Eggeler, A. Ludwig, Identification of quaternary shape memory alloys with near-zero thermal hysteresis and unprecedented functional stability, Adv. Funct. Mater. 20 (2010) 1917-1923.

[16] Y. Song, X. Chen, V. Dabade, T.W. Shield, R.D. James, Enhanced reversibility and unusual microstructure of a phase-transforming material, Nature. 502 (2013) 85-8. 
[17] C. Chluba, W. Ge, R. Lima de Miranda, J. Strobel, L. Kienle, E. Quandt, M. Wuttig, Ultralow-fatigue shape memory alloy films, Science. 348 (2015) 10041007.

[18] W.-F. Rao, M. Wuttig, A.G. Khachaturyan, Giant Nonhysteretic Responses of Two-Phase Nanostructured Alloys, Phys. Rev. Lett. 106 (2011) 105703.

[19] D. Wang, S. Hou, Y. Wang, X. Ding, S. Ren, X. Ren, Y. Wang, Superelasticity of slim hysteresis over a wide temperature range by nanodomains of martensite, Acta Mater. 66 (2014) 349-359.

[20] D. Wang, Z. Zhang, J. Zhang, Y. Zhou, Y. Wang, X. Ding, Y. Wang, X. Ren, Strain glass in Fe-doped Ti-Ni, Acta Mater. 58 (2010) 6206-6215.

[21] Y. Zhou, D. Xue, X. Ding, Y. Wang, J. Zhang, Z. Zhang, D. Wang, K. Otsuka, J. Sun, X. Ren, Strain glass in doped Ti50(Ni50-xDx) $(\mathrm{D}=\mathrm{Co}, \mathrm{Cr}, \mathrm{Mn})$ alloys: Implication for the generality of strain glass in defect-containing ferroelastic systems, Acta Mater. 58 (2010) 5433-5442.

[22] Y. Ji, X. Ding, T. Lookman, K. Otsuka, X. Ren, Heterogeneities and strain glass behavior: Role of nanoscale precipitates in low-temperature-aged Ti48.7Ni51.3 alloys, Phys. Rev. B. 87 (2013) 104110.

[23] S. Sarkar, X. Ren, K. Otsuka, Evidence for Strain Glass in the FerroelasticMartensitic System Ti50-xNi50+x, Phys. Rev. Lett. 95 (2005) 205702.

[24] P. Entel, M.E. Gruner, D. Comtesse, V. V. Sokolovskiy, V.D. Buchelnikov, Interacting magnetic cluster-spin glasses and strain glasses in $\mathrm{Ni}-\mathrm{Mn}$ based Heusler structured intermetallics, Phys. Status Solidi. 251 (2014) 2135-2148.

[25] J. Liu, M. Jin, C. Ni, Y. Shen, G. Fan, Z. Wang, Y. Zhang, C. Li, Z. Liu, X. Jin, Strain glassy behavior and premartensitic transition in Au7Cu5Al4 alloy, Phys. Rev. B. 84 (2011) 140102.

[26] D. Wang, D. Lv, Y. Gao, Y. Wang, X. Ren, Y. Wang, Defect strength and strain glass state in ferroelastic systems, J. Alloys Compd. 661 (2016) 100-109.

[27] Z. Zhang, X. Ding, J. Sun, T. Suzuki, T. Lookman, K. Otsuka, X. Ren, Nonhysteretic Superelasticity of Shape Memory Alloys at the Nanoscale, Phys. Rev. Lett. 111 (2013) 145701.

[28] W. Tang, Thermodynamic study of the low-temperature phase B19' and the martensitic transformation in near-equiatomic Ti-Ni shape memory alloys, Metall. Mater. Trans. A. 28 (1997) 537-544.

[29] J. Frenzel, E.P. George, A. Dlouhy, C. Somsen, M.F.X. Wagner, G. Eggeler, Influence of $\mathrm{Ni}$ on martensitic phase transformations in NiTi shape memory alloys, Acta Mater. 58 (2010) 3444-3458.

[30] J. Frenzel, A. Wieczorek, I. Opahle, B. Maaß, R. Drautz, G. Eggeler, On the effect of alloy composition on martensite start temperatures and latent heats in Ni-Ti-based shape memory alloys, Acta Mater. 90 (2015) 213-231.

[31] H.Y. Kim, Y. Ikehara, J.I. Kim, H. Hosoda, S. Miyazaki, Martensitic transformation, shape memory effect and superelasticity of $\mathrm{Ti}-\mathrm{Nb}$ binary alloys, Acta Mater. 54 (2006) 2419-2429. 
[32] Y.L. Hao, S.J. Li, S.Y. Sun, R. Yang, Effect of Zr and Sn on Young's modulus and superelasticity of Ti-Nb-based alloys, Mater. Sci. Eng. A. 441 (2006) 112118.

[33] W.F. Ho, C.P. Ju, J.H. Lin, Structure and properties of cast binary Ti-Mo alloys, Biomaterials. 20 (1999) 2115-22.

[34] T. Inamura, J.I. Kim, H.Y. Kim, H. Hosoda, K. Wakashima, S. Miyazaki, Composition dependent crystallography of $\alpha$ "-martensite in Ti-Nb-based $\beta$ titanium alloy, Philos. Mag. 87 (2007) 3325-3350.

[35] S.D. Prokoshkin, A. V. Korotitskiy, V. Brailovski, S. Turenne, I.Y. Khmelevskaya, I.B. Trubitsyna, On the lattice parameters of phases in binary Ti-Ni shape memory alloys, Acta Mater. 52 (2004) 4479-4492.

[36] Y.L. Hao, H.L. Wang, T. Li, J.M. Cairney, A.V. Ceguerra, Y.D. Wang, D. Wang, Y. Wang, H.L. Zhou, Q. Zhang, S.J. Li, R. Yang, Superelasticity and Tunable Thermal Expansion across a Wide Temperature Range, J. Mater. Sci. Technol. 32 (2016) 705-709.

[37] D.L. Moffat, U.R. Kattner, The stable and metastable Ti-Nb phase diagrams, Metall. Trans. A. 19 (1988) 2389-2397.

[38] K. Tsuchiya, H. Sato, S. Edo, K. Marukawa, M. Umemoto, Effect of aging on martensitic transformation in $\gamma-\mathrm{MnCu}$ alloy, Mater. Sci. Eng. A. 285 (2000) 353-356.

[39] F. Yin, Y. Ohsawa, A. Sato, K. Kawahara, Phase decomposition of the $\gamma$ phase in a Mn-30 at.\% Cu alloy during aging, Acta Mater. 48 (2000) 1273-1282.

[40] O. Lyon, C. Severac, C. Servant, Spinodal decomposition and isothermal $\omega$ phase formation in a Ti-Nb alloy, investigated by small-angle X-ray scattering, Philos. Mag. A. 48 (1983) 825-839.

[41] J.L. Murray, The Ti-V (Titanium-Vanadium) system, Bull. Alloy Phase Diagrams. 2 (1981) 48-55.

[42] F. Findik, Improvements in spinodal alloys from past to present, Mater. Des. 42 (2012) 131-146.

[43] J.E. Hilliard, Spinodal decomposition, in: H.I. Aaronson (Ed.), Phase Transform., American Society of Metals, Materials Park, OH, 1970: pp. 479560 .

[44] S. Hao, L. Cui, D. Jiang, X. Han, Y. Ren, J. Jiang, Y. Liu, Z. Liu, S. Mao, Y. Wang, Y. Li, X. Ren, X. Ding, S. Wang, C. Yu, X. Shi, M. Du, F. Yang, Y. Zheng, Z. Zhang, X. Li, D.E. Brown, J. Li, A Transforming Metal Nanocomposite with Large Elastic Strain, Low Modulus, and High Strength, Science. 339 (2013) 1191-1194.

[45] W.G. Burgers, On the process of transition of the cubic-body-centered modification into the hexagonal-close-packed modification of zirconium, Physica. 1 (1934) 561-586.

[46] J. Liu, Y.Y. Wang, Y.-L. Hao, Y.Y. Wang, Z. Nie, D. Wang, Y. Ren, Z. Lu, J. Wang, H. Wang, X. Hui, N. Lu, M.J. Kim, R. Yang, New intrinsic mechanism on gum-like superelasticity of multifunctional alloys, Sci. Rep. 3 (2013) 2156. 
[47] T. Hahn, Graphical symbols for symmetry elements in one, two and three dimensions, Int. Tables Crystallogr. Vol. A Space-Gr. Symmetry. 2 (2006) $7-$ 11 .

[48] Y. Gao, R. Shi, J.-F. Nie, S.A. Dregia, Y. Wang, Group theory description of transformation pathway degeneracy in structural phase transformations, Acta Mater. 109 (2016) 353-363.

[49] T. Ahmed, H.J. Rack, Martensitic transformations in Ti-(16-26 at\%) Nb alloys, J. Mater. Sci. 31 (1996) 4267-4276.

[50] Y. Zheng, R.E.A. Williams, S. Nag, R. Banerjee, H.L. Fraser, D. Banerjee, The effect of alloy composition on instabilities in the $\beta$ phase of titanium alloys, Scr. Mater. 116 (2016) 49-52.

[51] E.G. Obbard, Y.L. Hao, T. Akahori, R.J. Talling, M. Niinomi, D. Dye, R. Yang, Mechanics of superelasticity in Ti-30Nb-(8-10)Ta-5Zr alloy, Acta Mater. 58 (2010) 3557-3567.

[52] Y. Gao, N. Zhou, D. Wang, Y. Wang, Pattern formation during cubic to orthorhombic martensitic transformations in shape memory alloys, Acta Mater. 68 (2014) 93-105.

[53] A. Pathak, S. Banumathy, R. Sankarasubramanian, A. K. Singh, Orthorhombic martensitic phase in Ti-Nb alloys: A first principles study, Comput. Mater. Sci. 83 (2014) 222-228.

[54] S. Banumathy, R.K. Mandal, A K. Singh, Structure of orthorhombic martensitic phase in binary Ti-Nb alloys, J. Appl. Phys. 106 (2009) 093518.

[55] E.W. Collings, A Sourcebook of Titanium Alloy Superconductivity, Springer US, Boston, MA, 1983.

[56] J.W. Cahn, J.E. Hilliard, Free Energy of a Nonuniform System. I. Interfacial Free Energy, J. Chem. Phys. 28 (1958) 258-267.

[57] E.M. Lifshitz, L.P. Pitaevskii, Statistical Physics, Part I, in: L.D. Landau, E.M. Lifshitz (Eds.), Course Theor. Phys., 3rd ed., Pergamon Press, Oxford, 1980.

[58] Y.A. Izyumov, V.N. Syromyatnikov, Phase Transitions and Crystal Symmetry, Kluwer Academic Publishers, Boston, 1990.

[59] P. Tolédano, V. Dmitriev, Reconstructive Phase Transitions, World Scientific, New Jersey, 1996.

[60] Y. Wang, A.G. Khachaturyan, Multi-scale phase field approach to martensitic transformations, Mater. Sci. Eng. A. 438-440 (2006) 55-63.

[61] D.R. Trinkle, R.G. Hennig, S.G. Srinivasan, D.M. Hatch, M.D. Jones, H.T. Stokes, R.C. Albers, J.W. Wilkins, New Mechanism for the $\alpha$ to $\omega$ Martensitic Transformation in Pure Titanium, Phys. Rev. Lett. 91 (2003) 25701.

[62] D.E. Jiang, E.A. Carter, Carbon dissolution and diffusion in ferrite and austenite from first principles, Phys. Rev. B. 67 (2003) 214103.

[63] Y. Wang, A.G. Khachaturyan, Three-dimensional field model and computer modeling of martensitic transformations, Acta Mater. 45 (1997) 759-773. 
[64] A.G. Khachaturyan, Theory of Structural Transformations in Solids, John Wiley \& Sons, New York, 1983.

[65] J.D. Gunton, M. San Miguel, P.S. Sahni, The Dynamics of First Order Phase Transitions, in: C. Domb, J.L. Lebowitz (Eds.), Phase Transitions Crit. Phenom., Academic Press, New York, 1983: pp. 267-466.

[66] Y. Lu, C. Wang, Y. Gao, R. Shi, X. Liu, Y. Wang, Microstructure map for selforganized phase separation during film deposition, Phys. Rev. Lett. 109 (2012) 086101.

[67] L.-Q. Chen, Phase-Field Models for Microstructure Evolution, Annu. Rev. Mater. Res. 32 (2002) 113-140.

[68] J. Langer, M. Bar-on, H. Miller, New computational method in the theory of spinodal decomposition, Phys. Rev. A. 11 (1975) 1417-1429.

[69] J. Cahn, On spinodal decomposition, Acta Metall. 9 (1961) 795-801.

[70] Y.W. Zhang, S.J. Li, E.G. Obbard, H. Wang, S.C. Wang, Y.L. Hao, R. Yang, Elastic properties of $\mathrm{Ti}-24 \mathrm{Nb}-4 \mathrm{Zr}-8 \mathrm{Sn}$ single crystals with bcc crystal structure, Acta Mater. 59 (2011) 3081-3090.

[71] G.B. Olson, M. Cohen, A general mechanism of martensitic nucleation: Part I. General concepts and the FCC $\rightarrow$ HCP transformation, Metall. Trans. A. 7 (1976) 1897-1904.

[72] C. Huang, D. Browne, S. McFadden, A phase-field simulation of austenite to ferrite transformation kinetics in low carbon steels, Acta Mater. 54 (2006) 1121.

[73] J. Khalil-Allafi, W.W. Schmahl, T. Reinecke, Order parameter evolution and Landau free energy coefficients for the B2↔R-phase transition in a NiTi shape memory alloy, Smart Mater. Struct. 14 (2005) S192-S196.

[74] G. Olson, M. Cohen, A general mechanism of martensitic nucleation: Part II. $\mathrm{FCC} \rightarrow \mathrm{BCC}$ and other martensitic transformations, Metall. Trans. A. 7 (1976) 1905-1914.

[75] N.T. Panagiotopoulos, A. Moreira Jorge, I. Rebai, K. Georgarakis, W.J. Botta, A.R. Yavari, Nanoporous titanium obtained from a spinodally decomposed $\mathrm{Ti}$ alloy, Microporous Mesoporous Mater. 222 (2016) 23-26.

[76] H.Y. Kim, S. Hashimoto, J. Il Kim, H. Hosoda, S. Miyazaki, Mechanical Properties and Shape Memory Behavior of Ti-Nb Alloys, Mater. Trans. 45 (2004) 2443-2448.

[77] Y.L. Hao, S.J. Li, S.Y. Sun, C.Y. Zheng, R. Yang, Elastic deformation behaviour of $\mathrm{Ti}-24 \mathrm{Nb}-4 \mathrm{Zr}-7.9 \mathrm{Sn}$ for biomedical applications, Acta Biomater. 3 (2007) 277-286.

[78] X. Ren, Strain glass and ferroic glass - Unusual properties from glassy nanodomains, Phys. Status Solidi. 251 (2014) 1982-1992.

[79] X. Ren, Y. Wang, Y. Zhou, Z. Zhang, D. Wang, G. Fan, K. Otsuka, T. Suzuki, Y. Ji, J, Zhang, Y. Tian, S. Hou, X. Ding, Strain glass in ferroelastic systems: Premartensitic tweed versus strain glass, Philos. Mag. 90 (2010) 141-157. 
[80] P.P. Rao, B.K. Agrawal, A.M. Rao, Studies on spinodal decomposition in Cu27Ni-2Cr alloy, J. Mater. Sci. 21 (1986) 3759-3766.

[81] O. Soriano-Vargas, E.O. Avila-Davila, V.M. Lopez-Hirata, N. Cayetano-Castro, J.L. Gonzalez-Velazquez, Effect of spinodal decomposition on the mechanical behavior of Fe-Cr alloys, Mater. Sci. Eng. A. 527 (2010) 2910-2914.

[82] J.C. Zhao, M.R. Notis, Ordering transformation and spinodal decomposition in Au-Ni alloys, Metall. Mater. Trans. a-Physical Metall. Mater. Sci. 30 (1999) 707-716.

[83] Y. Ni, A.G. Khachaturyan, From chessboard tweed to chessboard nanowire structure during pseudospinodal decomposition., Nat. Mater. 8 (2009) 410-414.

[84] Y. Gao, N. Zhou, F. Yang, Y. Cui, L. Kovarik, N. Hatcher, R. Noebe, M.J. Mills, Y. Wang, P-phase precipitation and its effect on martensitic transformation in (Ni,Pt)Ti shape memory alloys, Acta Mater. 60 (2012) 15141527.

[85] J. Khalil-Allafi, A. Dlouhy, G. Eggeler, Ni4Ti3-precipitation during aging of NiTi shape memory alloys and its influence on martensitic phase transformations, Acta Mater. 50 (2002) 4255-4274.

[86] V.C. Solomon, M. Nishida, Effect of Aging on Martensitic Transformation in Ti-Rich Ti-Pd Shape Memory Alloy, Mater. Trans. 43 (2002) 897-901.

[87] R. Shi, Y. Wang, Variant selection during $\alpha$ precipitation in Ti-6Al-4V under the influence of local stress - A simulation study, Acta Mater. 61 (2013) 60066024.

[88] L. Zhang, D. Wang, X. Ren, Y. Wang, A new mechanism for low and temperature-independent elastic modulus, Sci. Rep. 5 (2015) 11477.

[89] Y. Wang, J. Gao, H. Wu, S. Yang, X. Ding, D. Wang, X. Ren, Y. Wang, X. Song, J. Gao, Strain glass transition in a multifunctional $\beta$-type Ti alloy, Sci. Rep. 4 (2014) 3995.

[90] R.D. James, Z. Zhang, R.D. James, Z. Zhang, A Way to Search for Multiferroic Materials with "Unlikely" Combinations of Physical Properties, in: A. Planes, L. Manosa, A. Saxena (Eds.), Magn. Struct. Funct. Mater., Springer, Berlin, 2005: pp. 159-175.

\section{Figure captions:}

Fig. 1. (a) Lattice correspondence between $\beta$ (parent) and $\alpha^{\prime \prime}$ (martensite) phases. (b) Variations of $\mathrm{M}_{\mathrm{S}}$ and principle strains $\left(\lambda_{1}, \lambda_{2}\right.$ and $\left.\lambda_{3}\right)$ of the MT in TiNb with $\mathrm{Nb}$ concentration. The eigenvectors corresponding to the eigenstrains $\lambda_{1}, \lambda_{2}$ and $\lambda_{3}$ are 
parallel to the lattice vectors of the martensitic phase, i.e., $\mathbf{a}, \mathbf{b}$ and $\mathbf{c}$, respectively, shown in (a).

Fig. 2. Comparison between stress strain curves obtained respectively from the phase field simulation (solid curve) and experiment (dashed curve) [70].

Fig. 3. (a) $\mathrm{Nb}$ concentration modulation developed by spinodal decomposition in the parent phase at $773 \mathrm{~K}$ with different aging time. (b1)-(b5) one-dimensional $\mathrm{Nb}$ concentration profiles along the body-diagonal of the computation cells shown in (a1)-(a5). (c1)-(c5) are statistical distributions of voxels having certain $\mathrm{Nb}$ concentration in the computational cell of (a1)-(a5). (d1)-(d5) are the stress strain curves under uniaxial tension along $[100]_{\beta}$ of the computation cells shown in (a1)-(a5). (e1)-(e5) are the normalized volume fraction of martensite during cyclic loading of the computation cells shown in (a1)-(a5).

Fig. 4. Microstructure evolution during MT in a concentration modulated system during loading ((a)-(d), along $\left.[100]_{\beta}\right)$ and unloading ((e)-(h)) processes. The locations of the microstructural states in (a)-(h) on the SS curve are indicated by the red dots in (i). In (a)-(h), the parent phase is set to be transparent and different variants (V) of the martensite are plotted as iso-surfaces with different colors, as indicated in the color map at the bottom. (j) Nb-lean (green) and Nb-rich (orange) regions in the parent phase, where the green and orange iso-surfaces correspond to $\mathrm{Nb}$ concentration of 9.3 and 19at.\%, respectively. (k) and (l) are close-up observations of the growth and shrinkage of the martensitic particle indicated by the arrows during loading and unloading, respectively. The background in (k) and (l) shows the $\mathrm{Nb}$ concentration distribution in the parent phase around the martensitic particle.

Fig. 5. Interaction energy density between the tensile external load of $1 \mathrm{MPa}$ along the $[100]_{\beta}$ direction and different martensitic variants. Variants that have a negative interaction energy density are preferred by the external load.

Fig. 6. (a) Landau free energy curves of volume elements of different $\mathrm{Nb}$ concentrations in the spinodally decomposed parent phase at $300 \mathrm{~K} . \mathrm{C}_{1}-\mathrm{C}_{5}$ have $\mathrm{Nb}$ concentration of $9.3,12.5,14.8,16.8$ and 19.3 at. $\%$, respectively. The positions of $\mathrm{C}_{1^{-}}$ $\mathrm{C}_{5}$ are indicated in (b). (b) Spatial variation of stability of martensite characterized by 
the driving force of MT, $\Delta f_{c h}=f_{c h}$ (Martensite) $-f_{c h}$ (Austenite) (in $10^{7} \mathrm{~J} / \mathrm{m}^{2}$ ), as defined in (a).

Fig. 7. Microstructure evolution during MT of the concentration modulated system shown in Fig. 3(a3) upon cooling and heating. (a)-(d) are microstructural states corresponding to the cooling process, while (e)-(h) are microstructural states corresponding to the heating process. In (a)-(h), the parent phase is set to be transparent and different variants $(\mathrm{V})$ of the martensitic phase are plotted as isosurfaces with different colors. The locations of (a)-(h) on the volume-temperature curve are marked by the red dots in (i).

Fig. 8. Variation of (a) $\sigma_{\mathrm{Ms}}$, (b) tangent modulus and hysteresis with aging time. The value of $\sigma_{\mathrm{Ms}}$ is determined by the tangent method shown in the inset of (a). The tangent modulus is defined as the slope of the transformation hardening region as shown in the inset of (b). The hysteresis refers to the area enclosed by the SS hysteresis loop.

Fig. 9. Microstructure evolution during stress-induced MT in a system aged for reduced time $\mathrm{t}^{*}=7100$ (Fig. 3(a5)) with cyclic loading along $[100]_{\beta}$ direction. (a)-(e) are microstructural states found in the loading process, while (f)-(h) are microstructural states found in the unloading process. In (a)-(h), the parent phase (A) and different variants of the martensitic phase (V1-V6) are represented by different colors. The location of (a)-(h) on SS curve is indicated by the red dots in (i). The inset of (i) shows the CM in the parent phase.

Fig. 10. Stress strain curve during stress-induced $\mathrm{MT}$ in a system with a uniform $\mathrm{Nb}$ concentration of 8 at.\% obtained from the phase field simulation.

Fig. 11. Stress strain curve during stress-induced MT obtained by the phase field simulation for a system with $\mathrm{CM}$ having a wavelength of $\sim 6 \mathrm{~nm}$ and an average $\mathrm{Nb}$ concentration of 15 at. $\%$ 

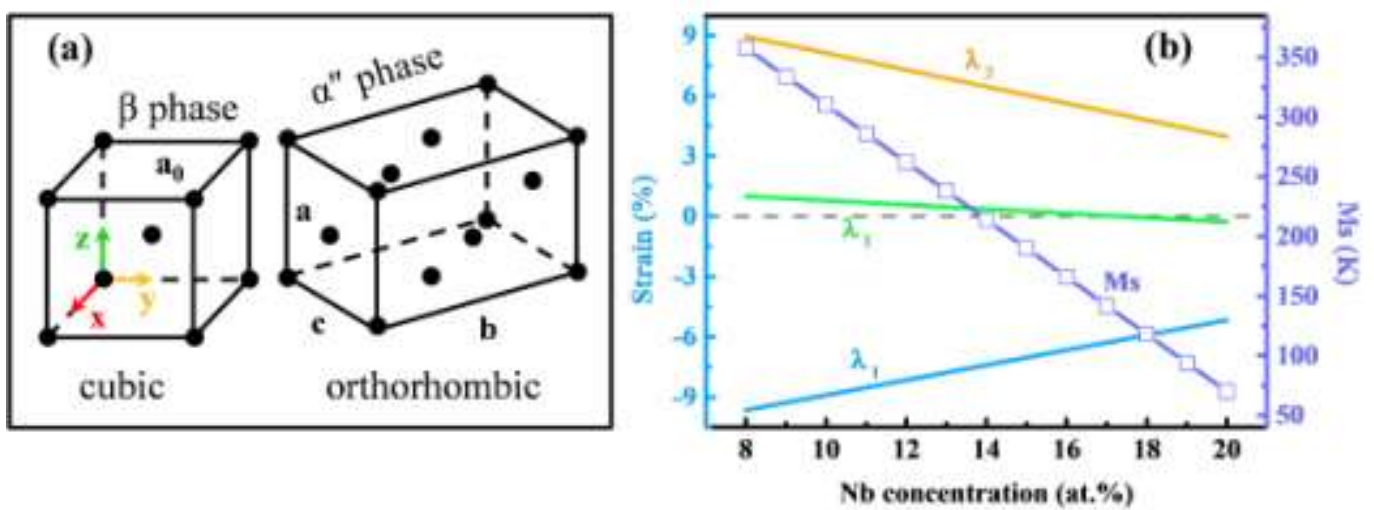

Fig. 1. (a) Lattice correspondence between $\beta$ (parent) and $\alpha^{\prime \prime}$ (martensite) phases. (b) Variations of $\mathrm{M}_{\mathrm{s}}$ and principle strains $\left(\lambda_{1}, \lambda_{2}\right.$ and $\left.\lambda_{3}\right)$ of the MT in TiNb with $\mathrm{Nb}$ concentration. The eigenvectors corresponding to the eigenstrains $\lambda_{1}, \lambda_{2}$ and $\lambda_{3}$ are parallel to the lattice vectors of the martensitic phase, i.e., $\mathbf{a}, \mathbf{b}$ and $\mathbf{c}$, respectively, shown in (a). 


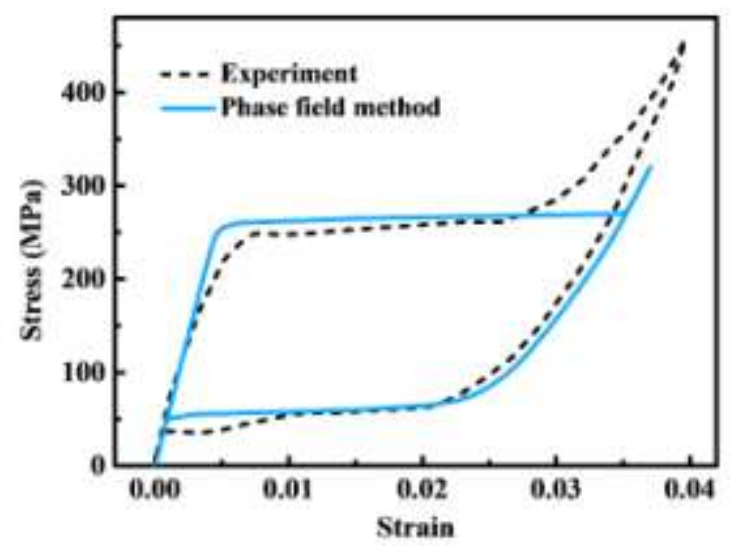

Fig. 2. Comparison between stress strain curves obtained respectively from the phase field simulation (solid curve) and experiment (dashed curve) [70]. 
(a)
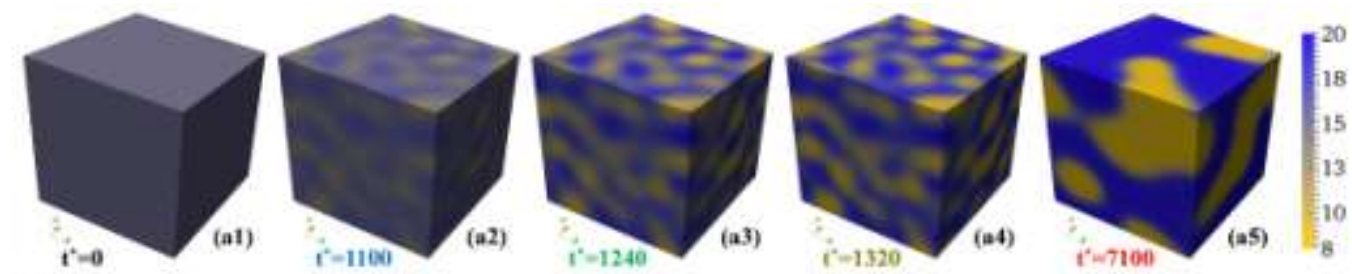

(b)

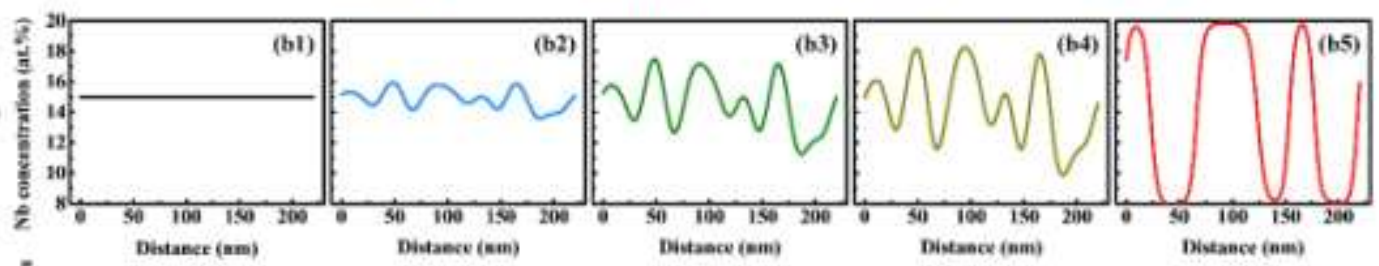

(c)
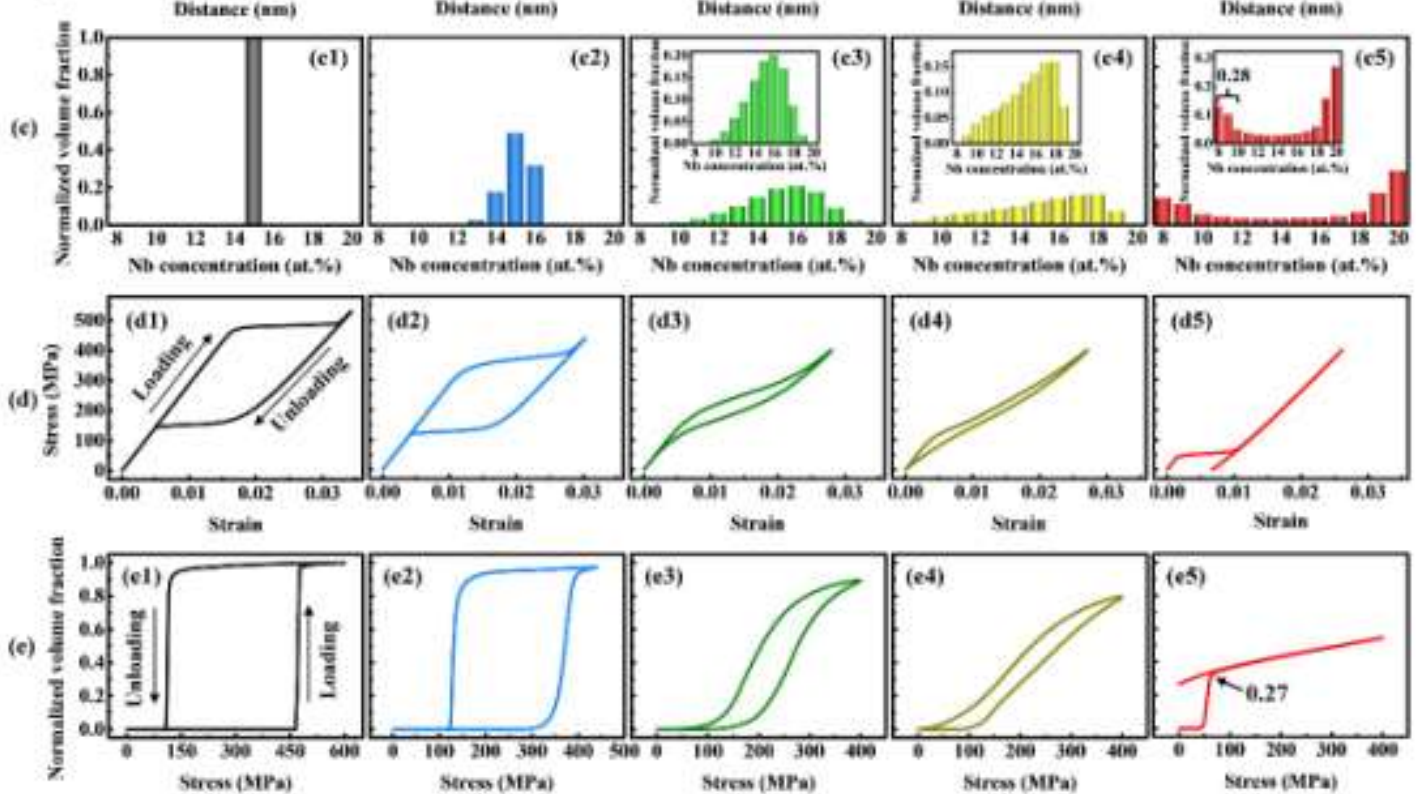

Fig. 3. (a) $\mathrm{Nb}$ concentration modulation developed by spinodal decomposition in the parent phase at $773 \mathrm{~K}$ with different aging time. (b1)-(b5) one-dimensional $\mathrm{Nb}$ concentration profiles along the body-diagonal of the computation cells shown in (a1)-(a5). (c1)-(c5) are statistical distributions of voxels having certain $\mathrm{Nb}$ concentration in the computational cell of (a1)-(a5). (d1)-(d5) are the stress strain curves under uniaxial tension along $[100]_{\beta}$ of the computation cells shown in (a1)-(a5). (e1)-(e5) are the normalized volume fraction of martensite during cyclic loading of the computation cells shown in (a1)-(a5). 

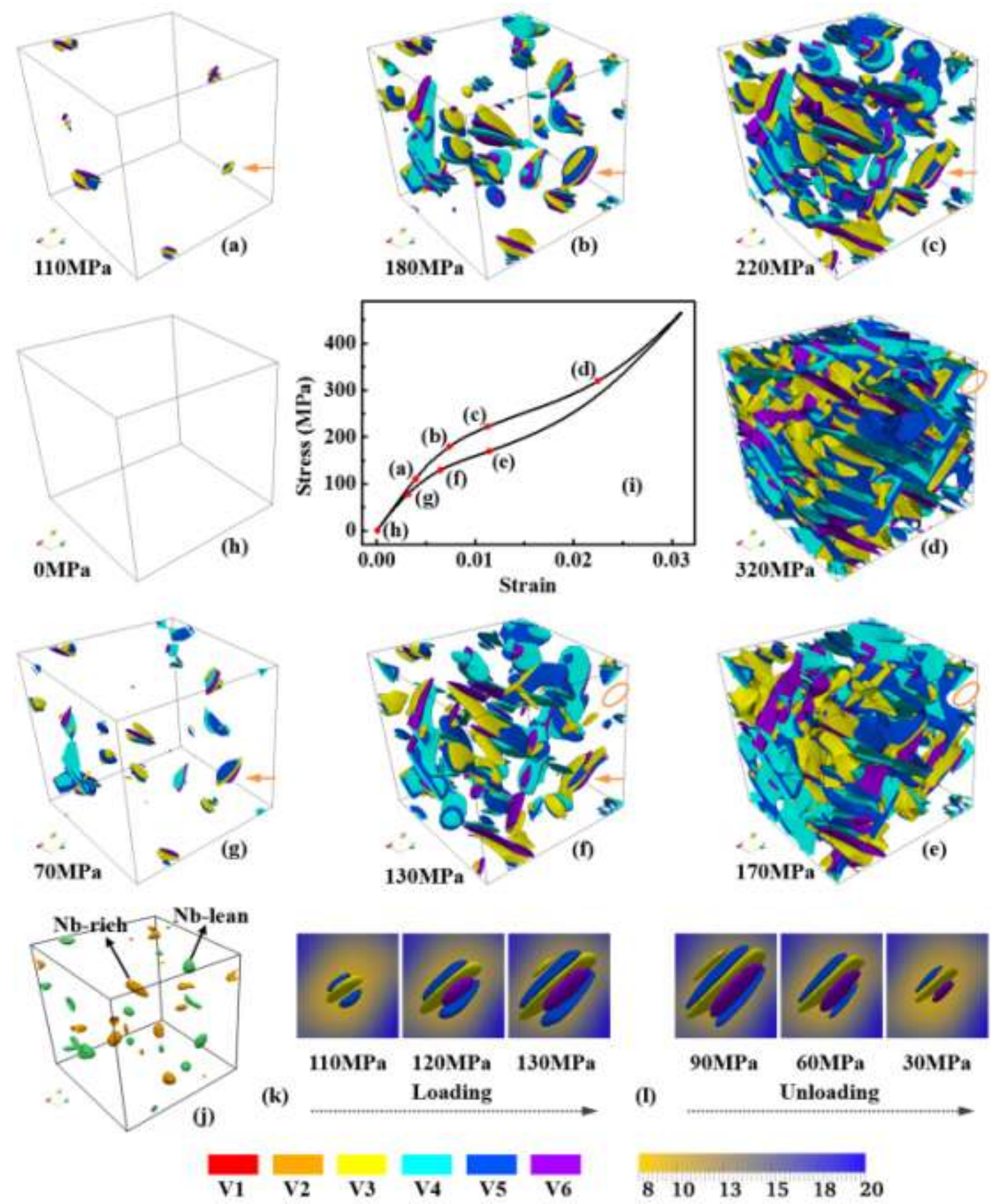

$\begin{array}{llllll}8 & 10 & 13 & 15 & 18 & 20\end{array}$

Fig. 4. Microstructure evolution during MT in a concentration modulated system during loading ((a)-(d), along $\left.[100]_{\beta}\right)$ and unloading ((e)-(h)) processes. The locations of the microstructural states in (a)-(h) on the SS curve are indicated by the red dots in (i). In (a)-(h), the parent phase is set to be transparent and different variants (V) of the martensite are plotted as iso-surfaces with different colors, as indicated in the color map at the bottom. (j) Nb-lean (green) and $\mathrm{Nb}$-rich (orange) regions in the parent phase, where the green and orange iso-surfaces correspond to $\mathrm{Nb}$ concentration of 9.3 and 
19at.\%, respectively. (k) and (l) are close-up observations of the growth and shrinkage of the martensitic particle indicated by the arrows during loading and unloading, respectively. The background in $(\mathrm{k})$ and (l) shows the $\mathrm{Nb}$ concentration distribution in the parent phase around the martensitic particle. 


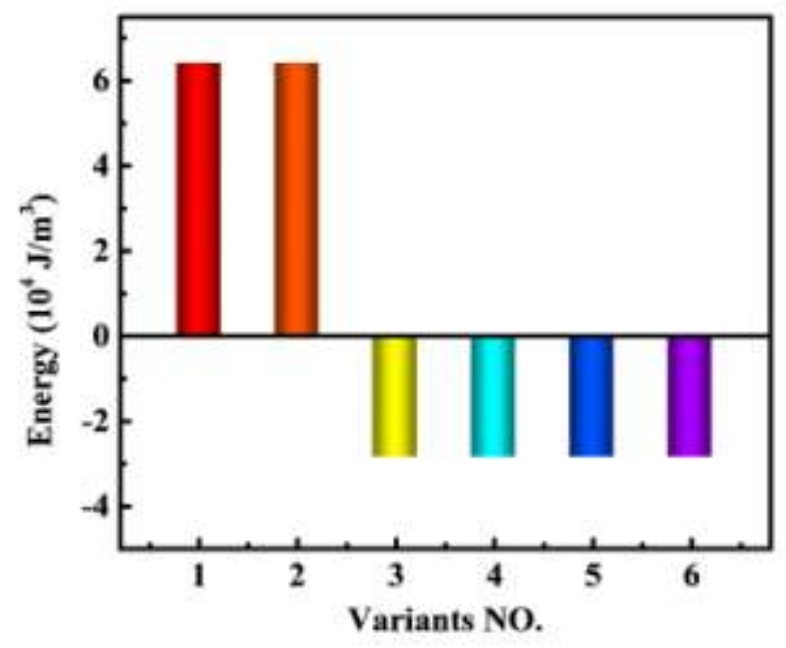

Fig. 5. Interaction energy density between the tensile external load of $1 \mathrm{MPa}$ along the $[100]_{\beta}$ direction and different martensitic variants. Variants that have a negative interaction energy density are preferred by the external load. 


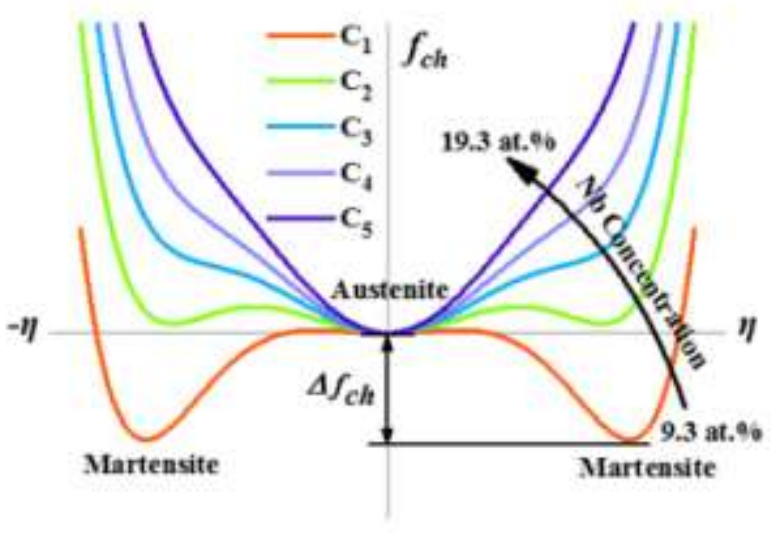

(a)

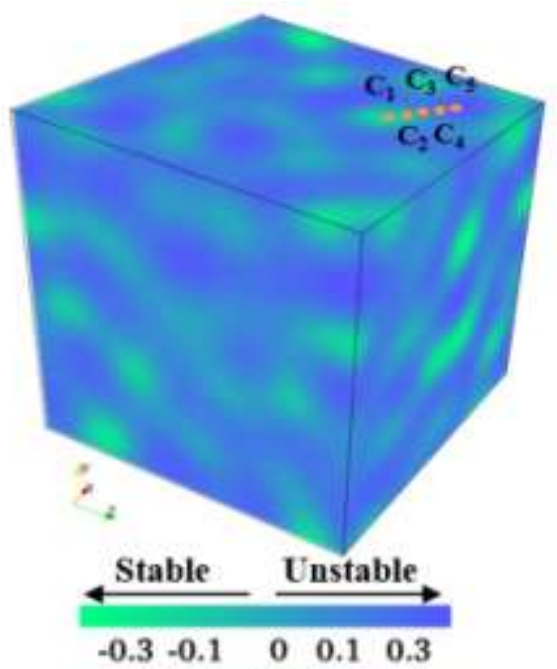

(b)

Fig. 6. (a) Landau free energy curves of volume elements of different $\mathrm{Nb}$ concentrations in the spinodally decomposed parent phase at $300 \mathrm{~K} . \mathrm{C}_{1}-\mathrm{C}_{5}$ have $\mathrm{Nb}$ concentration of $9.3,12.5,14.8,16.8$ and 19.3 at.\%, respectively. The positions of $\mathrm{C}_{1}-\mathrm{C}_{5}$ are indicated in (b). (b) Spatial variation of stability of martensite characterized by the driving force of MT, $\Delta f_{c h}=f_{c h}$ (Martensite) $-f_{c h}$ (Austenite) (in $10^{7} \mathrm{~J} / \mathrm{m}^{2}$ ), as defined in (a). 

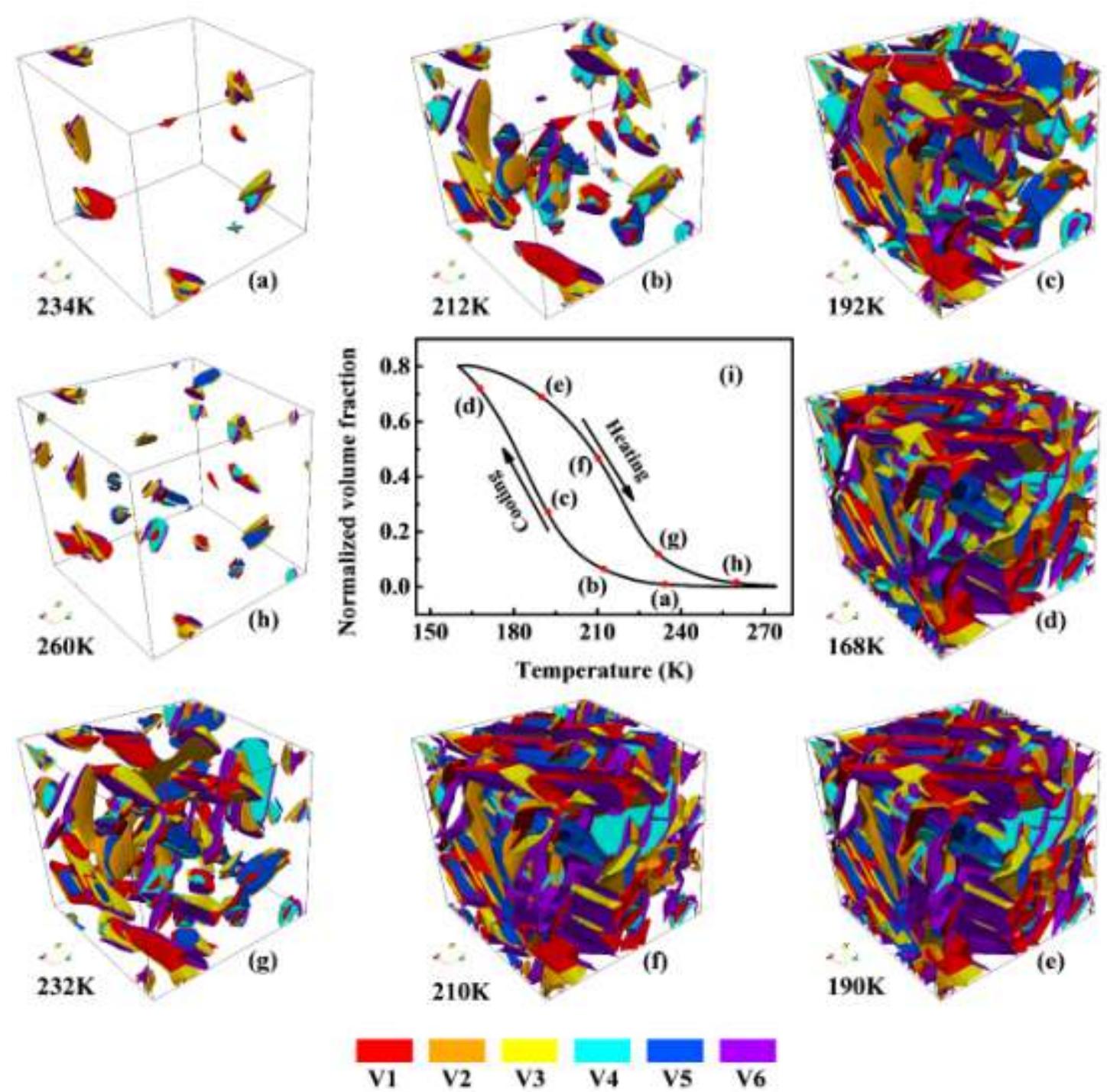

Fig. 7. Microstructure evolution during MT of the concentration modulated system shown in Fig. 3(a3) upon cooling and heating. (a)-(d) are microstructural states corresponding to the cooling process, while (e)-(h) are microstructural states corresponding to the heating process. In (a)-(h), the parent phase is set to be transparent and different variants $(\mathrm{V})$ of the martensitic phase are plotted as iso-surfaces with different colors. The locations of (a)-(h) on the volume-temperature curve are marked by the red dots in (i). 

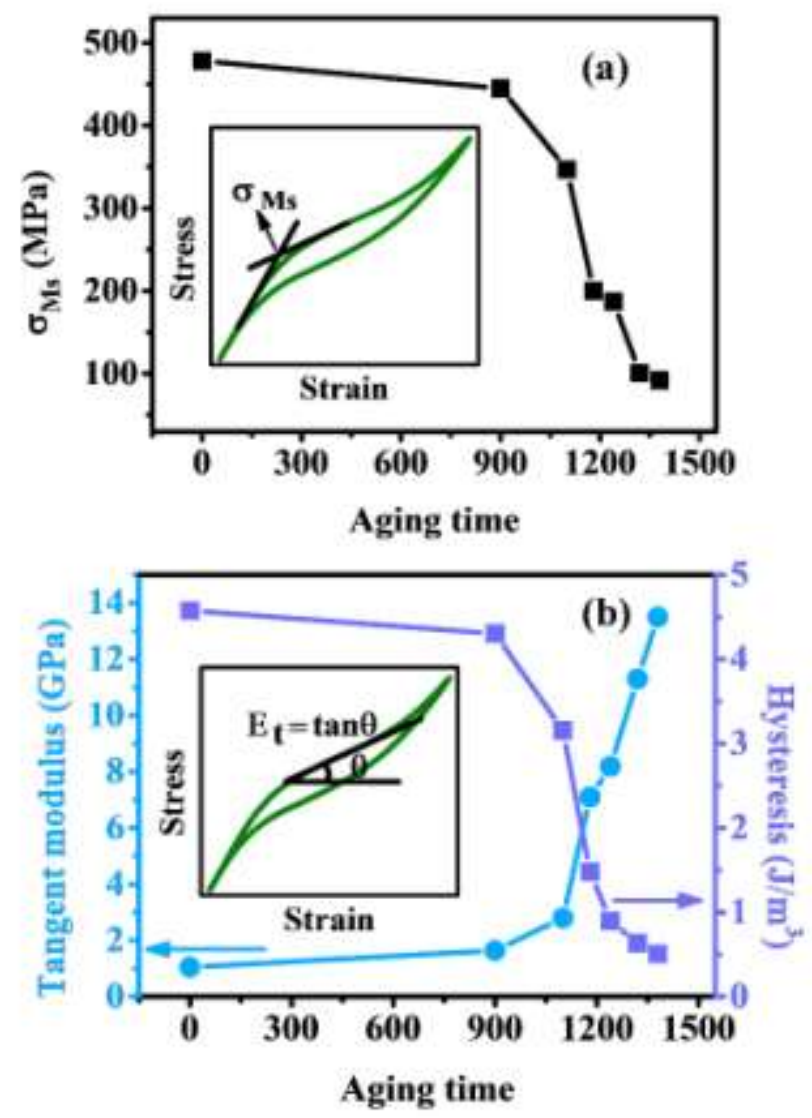

Fig. 8. Variation of (a) $\sigma_{\mathrm{Ms}}$, (b) tangent modulus and hysteresis with aging time. The value of $\sigma_{\mathrm{Ms}}$ is determined by the tangent method shown in the inset of (a). The tangent modulus is defined as the slope of the transformation hardening region as shown in the inset of (b). The hysteresis refers to the area enclosed by the SS hysteresis loop. 

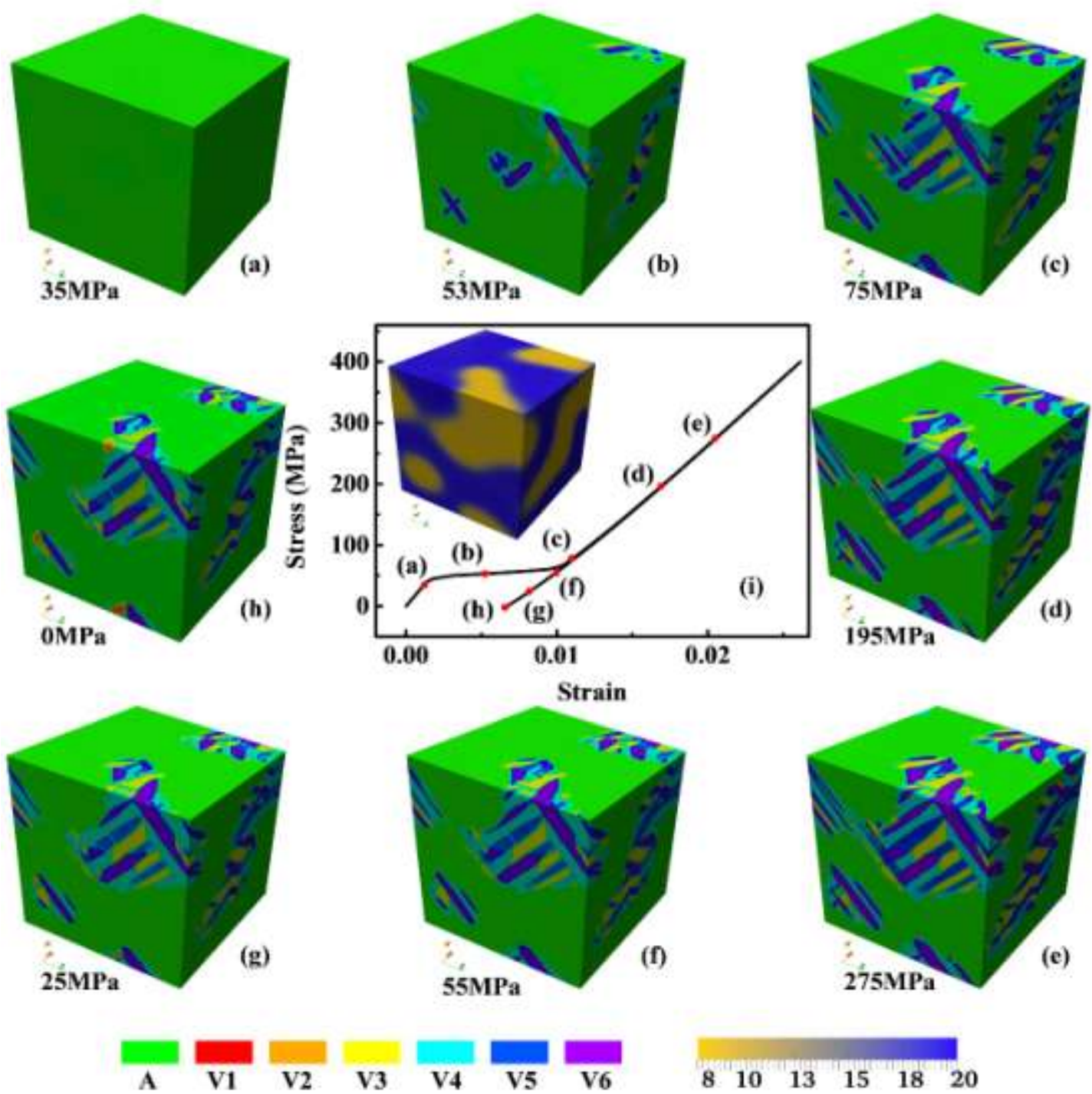

Fig. 9. Microstructure evolution during stress-induced MT in a system aged for reduced time $\mathrm{t}^{*}=7100$ (Fig. 3(a5)) with cyclic loading along $[100]_{\beta}$ direction. (a)-(e) are microstructural states found in the loading process, while (f)-(h) are microstructural states found in the unloading process. In (a)-(h), the parent phase (A) and different variants of the martensitic phase (V1-V6) are represented by different colors. The location of (a)-(h) on SS curve is indicated by the red dots in (i). The inset of (i) shows the $\mathrm{CM}$ in the parent phase. 


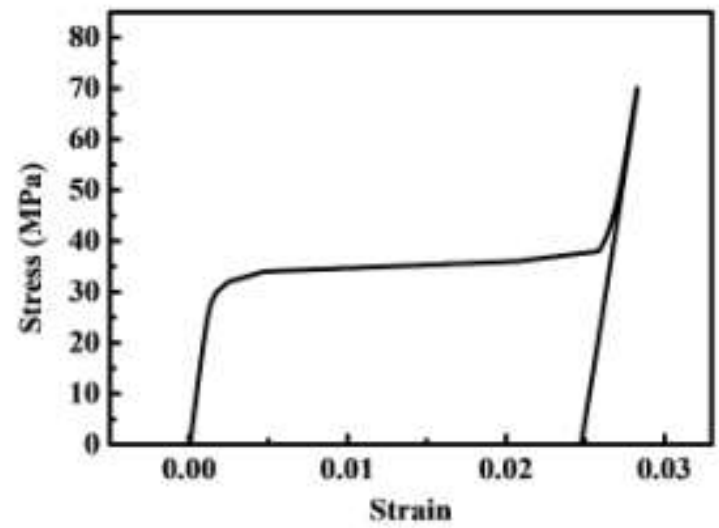

Fig. 10. Stress strain curve during stress-induced $\mathrm{MT}$ in a system with a uniform $\mathrm{Nb}$ concentration of 8 at.\% obtained from the phase field simulation. 


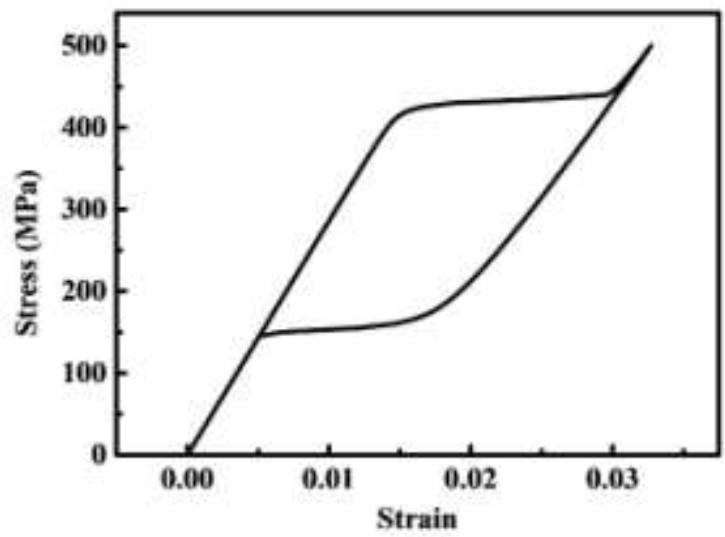

Fig. 11. Stress strain curve during stress-induced MT obtained by the phase field simulation for a system with $\mathrm{CM}$ having a wavelength of $\sim 6 \mathrm{~nm}$ and an average $\mathrm{Nb}$ concentration of 15 at.\% 


\section{Composition uniform system}

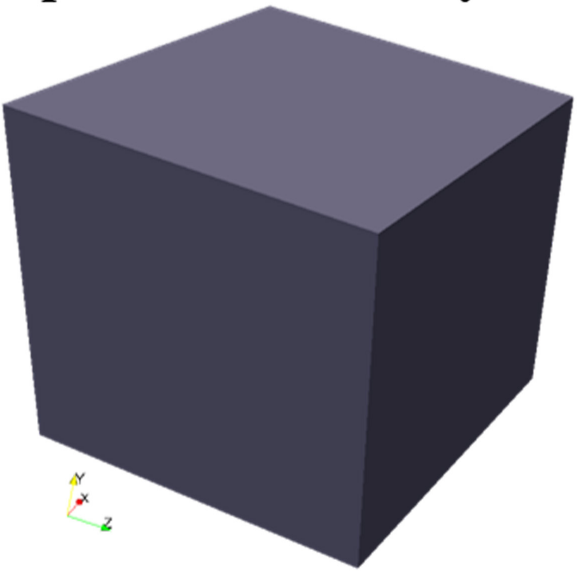

Concentration modulated system

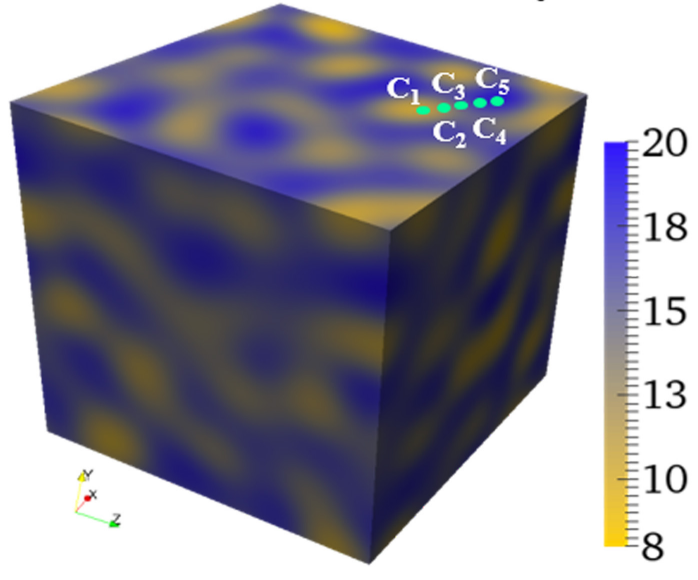

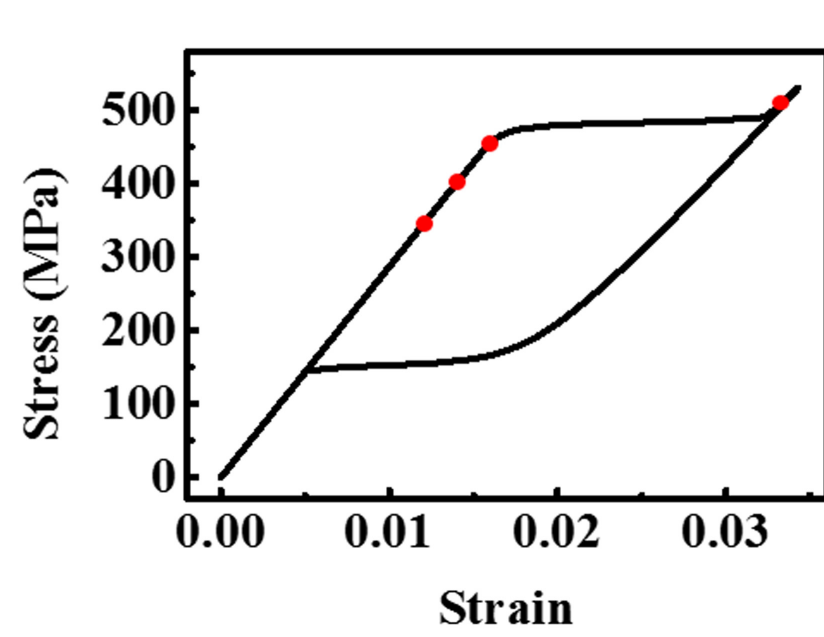

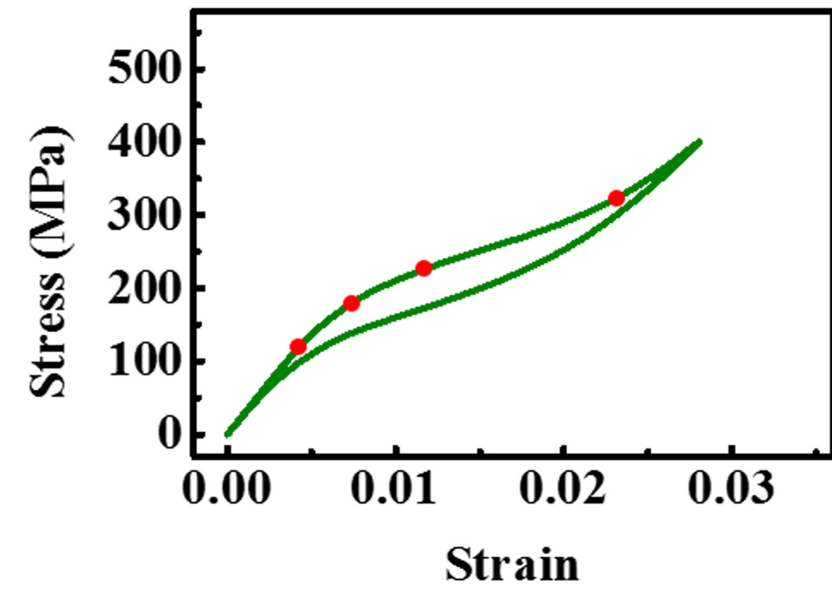

